\title{
Uniqueness of spaces pretangent to metric spaces at infinity
}

\author{
Oleksiy Dovgoshey, Viktoria Bilet
}

This paper dedicated to the memory of Professor Bogdan Bojarski

\begin{abstract}
We find the necessary and sufficient conditions under which an unbounded metric space $X$ has, at infinity, a unique pretangent space $\Omega_{\infty, \tilde{r}}^{X}$ for every scaling sequence $\tilde{r}$. In particular, it is proved that $\Omega_{\infty, \tilde{r}}^{X}$ is unique and isometric to the closure of $X$ for every logarithmic spiral $X$ and each $\tilde{r}$. It is also shown that the uniqueness of pretangent spaces to subsets of real line is closely related to "asymptotic asymmetry" of these subsets.
\end{abstract}

2010 MSC. 54E35.

Key words and phrases. Structure of metric space at infinity, logarithmic spiral, asymmetric set of real numbers, rescaling.

\section{Introduction}

The present paper deals with the so-called pretangent spaces to metric spaces at infinity introduced in $[4,5]$. Papers [4,5] as well as paper [6] describe the structure of metric spaces with finite pretangent spaces at infinity. The object of the present research is the metric spaces having, for given scaling sequence, unique pretangent spaces at infinity.

The main results of the paper are the following.

- Theorem 2.1 giving the necessary and sufficient conditions under which an unbounded metric space $X$ has a unique pretangent space $\Omega_{\infty, \tilde{r}}^{X}$ at infinity for every scaling sequence $\tilde{r}$.

- Theorem 3.1 which claims that each pretangent space to a logarithmic spiral at infinity is unique (for given scaling sequence), tangent, and isometric to the closure of this spiral.

Received 12.03.2019 
- Theorem 3.2 describing the uniqueness of $\Omega_{\infty, \tilde{r}}^{X}$ for the unbounded subgroups $X$ of the multiplicative group of nonzero complex numbers.

- Theorem 4.1 characterizing the subsets $X$ of $\mathbb{R}$ with unique $\Omega_{\infty, \tilde{r}}^{X}$ by symmetric properties of $X$.

The main results of the paper have natural infinitesimal analogs (see $[1,2])$.

Let us recall the definitions and denotations which will be used later.

Let $\tilde{r}=\left(r_{n}\right)_{n \in \mathbb{N}}$ be a sequence of positive real numbers tending to infinity, $\lim _{n \rightarrow \infty} r_{n}=\infty$. In what follows $\tilde{r}$ will be called a scaling sequence and, moreover, any formula of the form $\left(x_{n}\right)_{n \in \mathbb{N}} \subset A$ will mean that all elements of the sequence $\left(x_{n}\right)_{n \in \mathbb{N}}$ belong to the set $A$.

Let $(X, d)$ be an unbounded metric space.

Definition 1.1. Two sequences $\tilde{x}=\left(x_{n}\right)_{n \in \mathbb{N}} \subset X$ and $\tilde{y}=\left(y_{n}\right)_{n \in \mathbb{N}} \subset X$ are mutually stable with respect to the scaling sequence $\tilde{r}$ if there is a finite limit

$$
\lim _{n \rightarrow \infty} \frac{d\left(x_{n}, y_{n}\right)}{r_{n}}:=\tilde{d}_{\tilde{r}}(\tilde{x}, \tilde{y})=\tilde{d}(\tilde{x}, \tilde{y})
$$

Let $p \in X$. Denote by $\tilde{X}_{\infty}$ the set of all sequences $\tilde{x}=\left(x_{n}\right)_{n \in \mathbb{N}} \subset X$ for which $\lim _{n \rightarrow \infty} d\left(x_{n}, p\right)=\infty$ and write $\operatorname{Seq}(X, \tilde{r})$ for the set of all sequences $\tilde{x} \in \tilde{X}_{\infty}$ such that there is a finite limit

$$
\lim _{n \rightarrow \infty} \frac{d\left(x_{n}, p\right)}{r_{n}}:=\tilde{\tilde{d}}_{\tilde{r}}(\tilde{x}) .
$$

Definition 1.2. A subset $F$ of $\operatorname{Seq}(X, \tilde{r})$ is self-stable if any two $\tilde{x}, \tilde{y} \in F$ are mutually stable. $F$ is maximal self-stable if it is self-stable and, for arbitrary $\tilde{t} \in \operatorname{Seq}(X, \tilde{r})$, we have either $\tilde{t} \in F$ or there is $\tilde{x} \in F$ such that $\tilde{x}$ and $\tilde{t}$ are not mutually stable,

$$
\liminf _{n \rightarrow \infty} \frac{d\left(x_{n}, t_{n}\right)}{r_{n}}<\limsup _{n \rightarrow \infty} \frac{d\left(x_{n}, t_{n}\right)}{r_{n}} .
$$

The maximal self-stable subsets of $\operatorname{Seq}(X, \tilde{r})$ will be denoted by $\tilde{X}_{\infty, \tilde{r}}$.

Remark 1.1. The set $\tilde{X}_{\infty}$, the set $\operatorname{Seq}(X, \tilde{r})$ and its self-stable and maximal self-stable subsets are invariant under the choice of the point $p \in X$. If $\tilde{x} \in \operatorname{Seq}(X, \tilde{r})$ and $p, b \in X$, then, using the triangle inequality, we obtain

$$
\lim _{n \rightarrow \infty} \frac{d\left(x_{n}, p\right)}{r_{n}}=\lim _{n \rightarrow \infty} \frac{d\left(x_{n}, b\right)}{r_{n}} .
$$


Let $\tilde{X}_{\infty, \tilde{r}}$ be a maximal self-stable subset of $\operatorname{Seq}(X, \tilde{r})$. Consider the function $\tilde{d}: \tilde{X}_{\infty, \tilde{r}} \times \tilde{X}_{\infty, \tilde{r}} \rightarrow \mathbb{R}$ defined by (1.1). Obviously, $\tilde{d}$ is symmetric and nonnegative and $\tilde{d}(\tilde{x}, \tilde{x})=0$ holds for every $\tilde{x} \in \tilde{X}_{\infty, \tilde{r}}$. Moreover, the triangle inequality for $d$ gives us the triangle inequality for $\tilde{d}$,

$$
\tilde{d}(\tilde{x}, \tilde{y}) \leq \tilde{d}(\tilde{x}, \tilde{z})+\tilde{d}(\tilde{z}, \tilde{y}) .
$$

Hence $\left(\tilde{X}_{\infty, \tilde{r}}, \tilde{d}\right)$ is a pseudometric space.

Now we are ready to define the main object of our research.

Definition 1.3. Let $(X, d)$ be an unbounded metric space, let $\tilde{r}$ be a scaling sequence and let $\tilde{X}_{\infty, \tilde{r}}$ be a maximal self-stable subset of $\operatorname{Seq}(X, \tilde{r})$. The pretangent space to $(X, d)$ (at infinity, with respect to $\tilde{r}$ ) is the metric identification of the pseudometric space $\left(\tilde{X}_{\infty, \tilde{r}}, \tilde{d}\right)$.

Define a binary relation $\equiv$ on $\tilde{X}_{\infty, \tilde{r}}$ as

$$
(\tilde{x} \equiv \tilde{y}) \Leftrightarrow\left(\tilde{d}_{\tilde{r}}(\tilde{x}, \tilde{y})=0\right) .
$$

It is easy to prove that $\equiv$ is an equivalence relation on $\tilde{X}_{\infty, \tilde{r}}$. Write $\Omega_{\infty, \tilde{r}}^{X}$ for the set of equivalence classes generated by $\equiv$ and define the function $\rho: \Omega_{\infty, \tilde{r}}^{X} \times \Omega_{\infty, \tilde{r}}^{X} \rightarrow \mathbb{R}$ as

$$
\rho(\alpha, \beta):=\tilde{d}_{\tilde{r}}(\tilde{x}, \tilde{y}), \quad \tilde{x} \in \alpha \in \Omega_{\infty, \tilde{r}}^{X}, \quad \tilde{y} \in \beta \in \Omega_{\infty, \tilde{r}}^{X} .
$$

Using Theorem 15 [9, Chapter 4] it can be proved that $\rho$ is a well-defined metric on $\Omega_{\infty, \tilde{r}}^{X}$. The metric identification of $\left(\tilde{X}_{\infty, \tilde{r}}, \tilde{d}\right)$ is the metric space $\left(\Omega_{\infty, \tilde{r}}^{X}, \rho\right)$.

Let $\left(n_{k}\right)_{k \in \mathbb{N}} \subset \mathbb{N}$ be a strictly increasing sequence. Denote by $\tilde{r}^{\prime}$ the subsequence $\left(r_{n_{k}}\right)_{k \in \mathbb{N}}$ of the scaling sequence $\tilde{r}=\left(r_{n}\right)_{n \in \mathbb{N}}$ and, for every $\tilde{x}=\left(x_{n}\right)_{n \in \mathbb{N}} \in \tilde{X}_{\infty}$, write $\tilde{x}^{\prime}:=\left(x_{n_{k}}\right)_{k \in \mathbb{N}}$. It is clear that we have

$$
\left\{\tilde{x}^{\prime}: \tilde{x} \in \operatorname{Seq}(X, \tilde{r})\right\} \subseteq \operatorname{Seq}\left(X, \tilde{r}^{\prime}\right)
$$

and $\tilde{\tilde{d}}_{\tilde{r}^{\prime}}\left(\tilde{x}^{\prime}\right)=\tilde{\tilde{d}}_{\tilde{r}}(\tilde{x})$ holds for every $\tilde{x} \in \operatorname{Seq}(X, \tilde{r})$. Furthermore, if sequences $\tilde{x}, \tilde{y} \in \operatorname{Seq}(X, \tilde{r})$ are mutually stable w.r.t. $\tilde{r}$, then $\tilde{x}^{\prime}$ and $\tilde{y}^{\prime}$ are mutually stable w.r.t. $\tilde{r}^{\prime}$ and

$$
\tilde{d}_{\tilde{r}}(\tilde{x}, \tilde{y})=\tilde{d}_{\tilde{r}^{\prime}}\left(\tilde{x}^{\prime}, \tilde{y}^{\prime}\right)
$$

Consequently $\left\{\tilde{x}^{\prime}: \tilde{x} \in \tilde{X}_{\infty, \tilde{r}}\right\}$ is a self-stable subset of $\operatorname{Seq}\left(X, \tilde{r}^{\prime}\right)$ for every $\tilde{X}_{\infty, \tilde{r}}$. By Zorn's lemma there is $\tilde{X}_{\infty, \tilde{r}^{\prime}}$ such that

$$
\left\{\tilde{x}^{\prime}: \tilde{x} \in \tilde{X}_{\infty, \tilde{r}}\right\} \subseteq \tilde{X}_{\infty, \tilde{r}^{\prime}} \subseteq \operatorname{Seq}\left(X, \tilde{r}^{\prime}\right) .
$$


Let us denote by $\varphi_{\tilde{r}^{\prime}}$ the mapping from $\tilde{X}_{\infty, \tilde{r}}$ to $\tilde{X}_{\infty, \tilde{r}^{\prime}}$ with $\varphi_{\tilde{r}^{\prime}}(\tilde{x})=\tilde{x}^{\prime}$. It follows from (1.4) that, after corresponding metric identifications, the mapping $\varphi_{\tilde{r}^{\prime}}$ passes to an isometric embedding $\mathrm{em}^{\prime}: \Omega_{\infty, \tilde{r}}^{X} \rightarrow \Omega_{\infty, \tilde{r}^{\prime}}^{X}$ such that the diagram

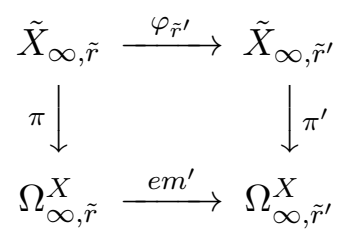

is commutative. Here $\pi$ and $\pi^{\prime}$ are the natural projections,

$\pi(\tilde{x})=\left\{\tilde{y} \in \tilde{X}_{\infty, \tilde{r}}: \tilde{d}_{\tilde{r}}(\tilde{x}, \tilde{y})=0\right\}$ and $\pi^{\prime}(\tilde{t})=\left\{\tilde{y} \in \tilde{X}_{\infty, \tilde{r}^{\prime}}: \tilde{d}_{\tilde{r}^{\prime}}(\tilde{t}, \tilde{y})=0\right\}$.

Definition 1.4. Let $(X, d)$ be an unbounded metric space and let $\tilde{r}$ be a scaling sequence. A pretangent $\Omega_{\infty, \tilde{r}}^{X}$ is tangent if em $\mathrm{em}^{\prime}: \Omega_{\infty, \tilde{r}}^{X} \rightarrow \Omega_{\infty, \tilde{r}^{\prime}}^{X}$ is surjective for every $\Omega_{\infty, \tilde{r}^{\prime}}^{X}$.

Remark 1.2. It can be proved that the following statements are equivalent.

- The metric space $\Omega_{\infty, \tilde{r}}^{X}$ is tangent.

- The mapping $\mathrm{em}^{\prime}: \Omega_{\infty, \tilde{r}}^{X} \rightarrow \Omega_{\infty, \tilde{r}^{\prime}}^{X}$ is an isometry for every $\Omega_{\infty, \tilde{r}^{\prime}}^{X}$.

- The set $\left\{\tilde{x}^{\prime}: \tilde{x} \in \tilde{X}_{\infty, \tilde{r}}\right\}$ is a maximal self-stable subset of the set $\operatorname{Seq}\left(X, \tilde{r}^{\prime}\right)$ for every $\tilde{r}^{\prime}$.

- The mapping $\varphi_{\tilde{r}^{\prime}}: \tilde{X}_{\infty, \tilde{r}} \rightarrow \tilde{X}_{\infty, \tilde{r}^{\prime}}$ is onto for every $\tilde{X}_{\infty, \tilde{r}^{\prime}}$.

\section{General criterion of uniqueness}

We start from an example of a metric space having the unique pretangent space for every scaling sequence.

Proposition 2.1. Let $X=\mathbb{R}^{+}=[0, \infty)$ be the set of all non-negative real numbers with the usual metric $d(x, y)=|x-y|$ and let $\tilde{r}=\left(r_{n}\right)_{r_{n} \in \mathbb{N}}$ be an arbitrary scaling sequence. Then the following statements hold for every maximal self-stable set $\tilde{X}_{\infty, \tilde{r}}$.

(i) The equality $\tilde{X}_{\infty, \tilde{r}}=\operatorname{Seq}(X, \tilde{r})$ holds. In particular, a sequence $\tilde{x} \in \tilde{X}_{\infty}$ belongs to $\tilde{X}_{\infty, \tilde{r}}$ if and only if there is $c \geq 0$ such that

$$
\tilde{\tilde{d}}_{\tilde{r}}(\tilde{x})=\lim _{n \rightarrow \infty} \frac{x_{n}}{r_{n}}=c .
$$


(ii) Let $\tilde{x}, \tilde{y} \in \tilde{X}_{\infty, \tilde{r}}$. Then the equality $\tilde{d}_{\tilde{r}}(\tilde{x}, \tilde{y})=0$ holds if and only if

$$
\lim _{n \rightarrow \infty} \frac{x_{n}}{r_{n}}=\lim _{n \rightarrow \infty} \frac{y_{n}}{r_{n}} .
$$

(iii) The pretangent space $\Omega_{\infty, \tilde{r}}^{X}$ corresponding to $\tilde{X}_{\infty, \tilde{r}}$ is isometric to $\left(\mathbb{R}^{+},|\cdot, \cdot|\right)$ and tangent.

Proof. (i) The inclusion $\tilde{X}_{\infty, \tilde{r}} \subseteq \operatorname{Seq}(X, \tilde{r})$ is trivial. Suppose now that $\tilde{x}, \tilde{y} \in \operatorname{Seq}(X, \tilde{r})$. Then choosing $p=0$ in the definition of $\operatorname{Seq}(X, \tilde{r})$ we can find $c_{1}, c_{2} \in \mathbb{R}^{+}$such that

$$
\lim _{n \rightarrow \infty} \frac{x_{n}}{r_{n}}=c_{1}, \quad \lim _{n \rightarrow \infty} \frac{y_{n}}{r_{n}}=c_{2} .
$$

Consequently,

$$
\lim _{n \rightarrow \infty} \frac{\left|x_{n}-y_{n}\right|}{r_{n}}=\left|c_{1}-c_{2}\right|
$$

holds, so that $\tilde{x}$ and $\tilde{y}$ are mutually stable. It implies statement $(i)$.

(ii) Statement (ii) follows from statement (i) and (2.2).

(iii) Define a function $f: \Omega_{\infty, \tilde{r}}^{X} \rightarrow \mathbb{R}^{+}$by the rule: if $\beta \in \Omega_{\infty, \tilde{r}}^{X}$ and $\tilde{x} \in \beta$, then $f(\beta):=\lim _{n \rightarrow \infty} \frac{x_{n}}{r_{n}}$. Statements $(i),(i i)$ and limit relation (2.2) imply that $f$ is a well-defined isometry.

Let $\tilde{n}=\left(n_{k}\right)_{k \in \mathbb{N}}$ be a strictly increasing sequence of positive integer numbers and let $\tilde{r}^{\prime}=\left(r_{n_{k}}\right)_{k \in \mathbb{N}}$ be the corresponding subsequence of the scaling sequence $\tilde{r}$. Suppose $\tilde{x} \in \tilde{X}_{\infty, \tilde{r}^{\prime}}$. Then there is $b \geqslant 0$ such that

$$
\lim _{k \rightarrow \infty} \frac{x_{k}}{r_{n_{k}}}=b
$$

Define $\tilde{y} \subset X$ as

$$
y_{n}:= \begin{cases}x_{k} & \text { if } n=n_{k} \in\left\{n_{1}, n_{2}, \ldots\right\}, \\ b r_{n} & \text { if } n \notin\left\{n_{1}, n_{2}, \ldots\right\} \text { and } b>0, \\ \left(r_{n}\right)^{1 / 2} & \text { if } n \notin\left\{n_{1}, n_{2}, \ldots\right\} \text { and } b=0 .\end{cases}
$$

It is clear that $\tilde{y} \in \tilde{X}_{\infty}$ and $\tilde{y}^{\prime}=\left(y_{n_{k}}\right)_{k \in \mathbb{N}}=\tilde{x}$ and

$$
\lim _{k \rightarrow \infty} \frac{y_{n}}{r_{n}}=b
$$

By statement $(i), \tilde{y}$ belongs to $\tilde{X}_{\infty, \tilde{r}}$, i.e., $\Omega_{\infty, \tilde{r}}^{X}$ is tangent.

Statement $(i)$ of Proposition 2.1 implies the following property of the metric space $(X, d)=(\mathbb{R}, \mid \cdot, \cdot)$. 
- For every scaling sequence $\tilde{r}$ there exists a unique pretangent space $\Omega_{\infty, \tilde{r}}^{X}$

We will denote by $\mathfrak{A}$ the class of all unbounded metric spaces having this property.

Remark 2.1. The uniqueness of pretangent spaces for $(X, d) \in \mathfrak{U}$ is understood in the usual set-theoretic sense. Each metric space is a pair consisting of a set and a metric defined on the Cartesian square of this set. The metric $\rho$ defined on $\Omega_{\infty, \tilde{r}}^{X} \times \Omega_{\infty, \tilde{r}}^{X}$ (see (1.1)) is evidently unique. Thus the uniqueness of pretangent space $\left(\Omega_{\infty, \tilde{r}}^{X}, \rho\right)$ means the uniqueness of the set $\Omega_{\infty, \tilde{r}}^{X}$. The last set is the quotient set of $\tilde{X}_{\infty, \tilde{r}}$ generated by $\equiv($ see $(1.2))$. The equivalence relation $\equiv$ on $\tilde{X}_{\infty, \tilde{r}}$ is unique for given $\tilde{r}$. Consequently, $\Omega_{\infty, \tilde{r}}^{X}$ is unique if and only if $\tilde{X}_{\infty, \tilde{r}}$ is unique. Recall that the construction of the maximal self-stable sets $\tilde{X}_{\infty, \tilde{r}}$ is based on the choice of elements $\tilde{x} \in \operatorname{Seq}(X, \tilde{r})$. Thus the statement $(X, d) \in \mathfrak{A}$ claims that the pretangent spaces to $(X, d)$ at infinity does not depend on this choice.

Let $(X, d)$ be a metric space and let $p \in X$. For each pair of nonempty sets $C, D \subseteq X$, we write

$$
\Delta(C, D):=\sup \{d(x, y): x \in C, y \in D\} .
$$

In addition, for every $\varepsilon \in(0,1)$, we define the set $S_{\varepsilon}^{2}$ as

$$
S_{\varepsilon}^{2}:=\left\{(r, t) \in S p^{2}(X): r \neq 0 \neq t \text { and }\left|\frac{r}{t}-1\right| \geq \varepsilon\right\}
$$

where $S p^{2}(X)$ is the Cartesian square of $S p(X)=\{d(x, p): x \in X\}$.

Theorem 2.1. Let $(X, d)$ be an unbounded metric space and let $p$ be a point of $X$. Then $(X, d) \in \mathfrak{A}$ if and only if the following conditions are satisfied simultaneously.

(i) The limit relations

$$
\lim _{k \rightarrow 1} \limsup _{r \rightarrow \infty} \frac{\operatorname{diam}(A(p, r, k))}{r}=\lim _{r \rightarrow \infty} \frac{\operatorname{diam}(S(p, r))}{r}=0
$$

hold, where $r \in(0, \infty), k \in[1, \infty)$ and $A(p, r, k)$ is the annulus

$$
\left\{x \in X: \frac{r}{k} \leq d(x, p) \leq r k\right\}
$$

and $S(p, r)$ is the sphere $\{x \in X: d(x, p)=r\}$. 
(ii) Let $\varepsilon \in(0,1)$. If $\left(\left(q_{n}, t_{n}\right)\right)_{n \in \mathbb{N}} \subset S_{\varepsilon}^{2}$ and

$$
\lim _{n \rightarrow \infty} q_{n}=\lim _{n \rightarrow \infty} t_{n}=\infty
$$

and there is

$$
\lim _{n \rightarrow \infty} \frac{q_{n}}{t_{n}}=c_{0} \in[0, \infty],
$$

then there exists a finite limit

$$
\lim _{n \rightarrow \infty} \frac{\Delta\left(S\left(p, q_{n}\right), S\left(p, t_{n}\right)\right)}{\left|q_{n}-t_{n}\right|}:=\kappa_{0} .
$$

The following lemma is immediate from the definition of the pretangent spaces.

Lemma 2.1. Let $(X, d)$ be an unbounded metric space. Then the following statements are equivalent.

(i) $(X, d) \in \mathfrak{A}$.

(ii) For every $\tilde{r}$, there is $\tilde{X}_{\infty, \tilde{r}}$ such that

$$
\tilde{X}_{\infty, \tilde{r}}=\operatorname{Seq}(X, \tilde{r}) .
$$

(iii) Equality (2.7) holds for every $\tilde{r}$ and every $\tilde{X}_{\infty, \tilde{r}}$.

(iv) All $\tilde{x}, \tilde{y} \in \operatorname{Seq}(X, \tilde{r})$ are mutually stable for every $\tilde{r}$.

Suppose $(X, d)$ is a metric space. For all nonempty $A, B \subseteq X$ we define the distance from $A$ to $B$ as

$$
\inf \{d(x, y): x \in A, y \in B\}
$$

and denote it by $\delta(A, B)$. (See, for example, [11, Definition 2.7.1].)

Lemma 2.2. Let $(X, d)$ be an unbounded metric space, $p \in X$. If condition ( $i$ ) from Theorem 2.1 holds, then we have the equality

$$
\lim _{\substack{t, q \rightarrow \infty \\(t, q) \in S_{\varepsilon}^{2}}} \frac{\Delta(S(p, q), S(p, t))}{\delta(S(p, q), S(p, t))}=1
$$

for every $\varepsilon \in(0,1)$. 
Proof. Suppose there is $\varepsilon \in(0,1)$ such that $(2.8)$ does not hold. Then there exist two sequences $\left(S\left(p, q_{n}\right)\right)_{n \in \mathbb{N}}$ and $\left(S\left(p, t_{n}\right)\right)_{n \in \mathbb{N}}$ with

$$
\left(\left(t_{n}, q_{n}\right)\right)_{n \in \mathbb{N}} \subset S_{\varepsilon}^{2}
$$

and four sequences $\tilde{x}, \tilde{y}, \tilde{z}, \tilde{w} \in \tilde{X}_{\infty}$ such that

$$
x_{n}, z_{n} \in S\left(p, t_{n}\right) \quad \text { and } \quad y_{n}, w_{n} \in S\left(p, q_{n}\right),
$$

for every $n \in \mathbb{N}$, and

$$
\limsup _{n \rightarrow \infty} \frac{d\left(x_{n}, y_{n}\right)}{d\left(z_{n}, w_{n}\right)}>1
$$

Suppose also that condition (i) of Theorem 2.1 holds. Then we obtain

$$
\lim _{n \rightarrow \infty} \frac{d\left(x_{n}, z_{n}\right)}{t_{n}}=\lim _{n \rightarrow \infty} \frac{d\left(y_{n}, w_{n}\right)}{q_{n}}=0 .
$$

Note also that the relation $\left(t_{n}, q_{n}\right) \in S_{\varepsilon}^{2}$ and (2.3) imply that there is $\varepsilon_{1} \in(0, \varepsilon)$ such that the inequalities

$$
\left|q_{n}-t_{n}\right| \geq \varepsilon_{1} q_{n} \text { and }\left|t_{n}-q_{n}\right| \geq \varepsilon_{1} t_{n}
$$

hold for every $n \in \mathbb{N}$. Let us find the upper bound of the limits in (2.4). Write

$$
\eta_{n}:=\frac{d\left(x_{n}, z_{n}\right)}{t_{n}} \text { and } \xi_{n}:=\frac{d\left(y_{n}, w_{n}\right)}{q_{n}}
$$

for every $n \in \mathbb{N}$. The triangle inequality implies

$$
\begin{gathered}
\frac{d\left(x_{n}, y_{n}\right)}{d\left(z_{n}, w_{n}\right)} \leq \frac{d\left(x_{n}, z_{n}\right)}{d\left(z_{n}, w_{n}\right)}+\frac{d\left(z_{n}, w_{n}\right)}{d\left(z_{n}, w_{n}\right)}+\frac{d\left(w_{n}, y_{n}\right)}{d\left(z_{n}, w_{n}\right)} \\
\leq \eta_{n} \frac{t_{n}}{d\left(z_{n}, w_{n}\right)}+1+\xi_{n} \frac{q_{n}}{d\left(z_{n}, w_{n}\right)} .
\end{gathered}
$$

Using

$$
d\left(z_{n}, w_{n}\right) \geq\left|d\left(z_{n}, p\right)-d\left(w_{n}, p\right)\right|=\left|t_{n}-q_{n}\right|
$$

and (2.11) we obtain

$$
\frac{t_{n}}{d\left(z_{n}, w_{n}\right)} \leq \frac{t_{n}}{\left|t_{n}-q_{n}\right|} \leq \frac{1}{\varepsilon_{1}} \quad \text { and } \quad \frac{q_{n}}{d\left(z_{n}, w_{n}\right)} \leq \frac{q_{n}}{\left|t_{n}-q_{n}\right|} \leq \frac{1}{\varepsilon_{1}} .
$$

Thus,

$$
\limsup _{n \rightarrow \infty} \frac{d\left(x_{n}, y_{n}\right)}{d\left(z_{n}, w_{n}\right)} \leq 1+\frac{1}{\varepsilon_{1}} \limsup _{n \rightarrow \infty}\left(\eta_{n}+\xi_{n}\right) .
$$

The upper limit in the right is zero by (2.10). Consequently we have

$$
\limsup _{n \rightarrow \infty} \frac{d\left(x_{n}, y_{n}\right)}{d\left(z_{n}, w_{n}\right)} \leq 1,
$$

contrary to $(2.9)$. 
Proof Theorem 2.1. Assume $(X, d) \in \mathfrak{A}$. We need verify conditions $(i)$ and $(i i)$.

(i) Let us consider the function $f:[1, \infty) \rightarrow \mathbb{R}^{+}$with

$$
f(k):=k \limsup _{r \rightarrow \infty} \frac{\operatorname{diam}(A(p, r, k))}{r} .
$$

Since

$$
f(k)=\limsup _{r \rightarrow \infty} \frac{\operatorname{diam}\left(A\left(p, k \frac{r}{k}, k\right)\right)}{\frac{r}{k}}=\limsup _{t \rightarrow \infty} \frac{\operatorname{diam}(A(p, k t, k))}{t}
$$

and

$$
A(p, k t, k)=\left\{x \in X: t \leq d(x, p) \leq k^{2} t\right\},
$$

the function $f$ is increasing. We evidently have

$$
\frac{\operatorname{diam}(A(p, r, k))}{r} \leq \frac{2 r k}{r}=2 k
$$

for all $k \geq 1$ and $r>0$. Hence the double inequality

$$
0 \leq f(k) \leq 2 k^{2}
$$

holds. Consequently, there is a finite, positive limit

$$
\lim _{k \rightarrow 1} f(k):=c_{0} .
$$

It is clear that this limit equals $\Psi(1)$. Suppose that $c_{0}>0$ and set $\varepsilon \in\left(0, c_{0}\right)$. Then there is $k_{0}>1$ such that the double inequality

$$
c_{0}-\varepsilon<\limsup _{r \rightarrow \infty} \frac{\operatorname{diam}(A(p, r, k))}{r}<c_{0}+\varepsilon
$$

holds for every $k \in\left(1, k_{0}\right]$. Let $\left(k_{n}\right)_{n \in \mathbb{N}} \subset\left(1, k_{0}\right]$ be a strictly decreasing sequence such that

$$
\lim _{n \rightarrow \infty} k_{n}=1 .
$$

Double inequality (2.12) implies that there is a sequence $\tilde{r}=\left(r_{n}\right)_{n \in \mathbb{N}}$ such that $\lim _{n \rightarrow \infty} r_{n}=\infty$ and

$$
c_{0}-\varepsilon<\frac{\operatorname{diam}\left(A\left(p, r_{n}, k_{n}\right)\right)}{r_{n}}<c_{0}+\varepsilon
$$

for every $n \in \mathbb{N}$. It follows from (2.14) that there are $\tilde{x}, \tilde{y} \subset X$ such that

$$
x_{n}, y_{n} \in A\left(p, r_{n}, k_{n}\right) \text { and } \frac{d\left(x_{n}, y_{n}\right)}{r_{n}} \geq c_{0}-\varepsilon
$$


for every $n \in \mathbb{N}$. The definition of $A\left(p, r_{n}, k_{n}\right)$ and (2.15) imply that

$$
\frac{d\left(x_{n}, p\right)}{r_{n}}, \quad \frac{d\left(y_{n}, p\right)}{r_{n}} \in\left[\frac{1}{k_{n}}, k_{n}\right]
$$

for every $n \in \mathbb{N}$. Define a sequence $\tilde{z}=\left(z_{n}\right)_{n \in \mathbb{N}} \subset X$ by the rule

$$
z_{n}:= \begin{cases}x_{n} & \text { if } n \text { is even } \\ y_{n} & \text { if } n \text { is odd }\end{cases}
$$

Then it follows from (2.13), (2.16) and (2.17) that

$$
\lim _{n \rightarrow \infty} \frac{d\left(x_{n}, p\right)}{r_{n}}=\lim _{n \rightarrow \infty} \frac{d\left(z_{n}, p\right)}{r_{n}}=1 .
$$

Moreover, (2.14) and (2.16) imply

$$
\liminf _{n \rightarrow \infty} \frac{d\left(x_{n}, z_{n}\right)}{r_{n}}=\lim _{n \rightarrow \infty} \frac{d\left(x_{2 n}, z_{2 n}\right)}{r_{2 n}}=0,
$$

but

$$
\limsup _{n \rightarrow \infty} \frac{d\left(x_{n}, z_{n}\right)}{r_{n}}=\limsup _{n \rightarrow \infty} \frac{d\left(x_{2 n+1}, z_{2 n+1}\right)}{r_{2 n+1}} \geq c_{0}-\varepsilon>0 .
$$

Thus $\tilde{x}$ and $\tilde{p}$ are mutually stable, $\tilde{z}$ and $\tilde{p}$ are mutually stable but $\tilde{x}$ and $\tilde{z}$ are not mutually stable (w.r.t. the scaling sequence $\tilde{r}=\left(r_{n}\right)_{n \in \mathbb{N}}$ ). Hence, by Lemma 2.1 , we have $(X, d) \notin \mathfrak{A}$, contrary to the assumption.

(ii) Let $\left(\left(q_{n}, t_{n}\right)\right)_{n \in \mathbb{N}}$ be a sequence of elements of $S_{\varepsilon}^{2}$ such that $\lim _{n \rightarrow \infty} q_{n}=\lim _{n \rightarrow \infty} t_{n}=\infty$ and (2.5) hold. If $c_{0}=0$ or $c_{0}=\infty$ in (2.5), then it is clear that (2.6) holds with $\kappa_{0}=1$, so it is sufficient to set

$$
c_{0} \in(0, \infty) .
$$

Let $\tilde{x}, \tilde{y} \in \tilde{X}_{\infty}$ such that $d\left(p, x_{n}\right)=q_{n}, d\left(p, y_{n}\right)=t_{n}$ for every $n \in \mathbb{N}$ and

$$
\lim _{n \rightarrow \infty} \frac{d\left(x_{n}, y_{n}\right)}{\Delta\left(S\left(p, q_{n}\right), S\left(p, t_{n}\right)\right)}=1 .
$$

Let us consider the sequence $\tilde{q}=\left(q_{n}\right)_{n \in \mathbb{N}}$ as a scaling sequence. Conditions (2.5) and (2.18) imply that there is

$$
\tilde{\tilde{d}}_{\tilde{q}}(\tilde{y})=\lim _{n \rightarrow \infty} \frac{d\left(y_{n}, p\right)}{q_{n}}=\frac{1}{c_{0}}<\infty .
$$

Hence, by Lemma 2.1, there is a finite limit

$$
\tilde{d}_{\tilde{q}}(\tilde{x}, \tilde{y})=\lim _{n \rightarrow \infty} \frac{d\left(x_{n}, y_{n}\right)}{q_{n}} .
$$


Moreover, since, for every $n \in \mathbb{N},\left(q_{n}, t_{n}\right) \in S_{\varepsilon}^{2}$, we have $c_{0} \neq 1$. Now using (2.19) and (2.5) we obtain

$$
\begin{array}{r}
\lim _{n \rightarrow \infty} \frac{\Delta\left(S\left(p, q_{n}\right), S\left(p, t_{n}\right)\right)}{\left|q_{n}-t_{n}\right|}=\lim _{n \rightarrow \infty} \frac{d\left(x_{n}, y_{n}\right) \Delta\left(S\left(p, q_{n}\right), S\left(p, t_{n}\right)\right)}{q_{n}\left|1-\frac{t_{n}}{q_{n}}\right| d\left(x_{n}, y_{n}\right)} \\
=\lim _{n \rightarrow \infty} \frac{d\left(x_{n}, y_{n}\right)}{q_{n}} \lim _{n \rightarrow \infty} \frac{1}{\left|1-\frac{t_{n}}{q_{n}}\right|}=\frac{c_{0}}{\left|1-c_{0}\right|} \tilde{d}_{\tilde{q}}(\tilde{x}, \tilde{y}) .
\end{array}
$$

Condition (ii) is proved.

To prove that $(i)$ and $(i i)$ imply $(X, d) \in \mathfrak{A}$ suppose $(i)$ and (ii) are satisfied simultaneously. We must show that $\Omega_{\infty, \tilde{r}}^{X}$ is unique for every scaling sequence $\tilde{r}$. Let $\tilde{r}=\left(r_{n}\right)_{n \in \mathbb{N}}$ be an arbitrary scaling sequence and let $\tilde{x}, \tilde{y} \in \operatorname{Seq}(X, \tilde{r})$. By Lemma 2.1 it is sufficient to show that $\tilde{x}$ and $\tilde{y}$ are mutually stable. If $\tilde{\tilde{d}}_{\tilde{r}}(\tilde{x})=0$, then, by the triangle inequality,

$$
\limsup _{n \rightarrow \infty} \frac{d\left(x_{n}, y_{n}\right)}{r_{n}} \leq \tilde{\tilde{d}}_{\tilde{r}}(\tilde{x})+\tilde{\tilde{d}}_{\tilde{r}}(\tilde{y})=\tilde{\tilde{d}}_{\tilde{r}}(\tilde{y})
$$

and

$$
\liminf _{n \rightarrow \infty} \frac{d\left(x_{n}, y_{n}\right)}{r_{n}} \geq \tilde{\tilde{d}}_{\tilde{r}}(\tilde{y})-\tilde{\tilde{d}}_{\tilde{r}}(\tilde{x})=\tilde{\tilde{d}}_{\tilde{r}}(\tilde{y}) .
$$

Consequently

$$
\tilde{d}_{\tilde{r}}(\tilde{x}, \tilde{y})=\lim _{n \rightarrow \infty} \frac{d\left(x_{n}, y_{n}\right)}{r_{n}}=\tilde{\tilde{d}}_{\tilde{r}}(\tilde{y}),
$$

holds. Thus $\tilde{x}$ and $\tilde{y}$ are mutually stable. The case $\tilde{\tilde{d}}_{\tilde{r}}(\tilde{y})=0$ is similar. Hence, without loss of generality, we may assume that

$$
\tilde{\tilde{d}}_{\tilde{r}}(\tilde{y}) \neq 0 \neq \tilde{\tilde{d}}_{\tilde{r}}(\tilde{x}) .
$$

Consider first the case

$$
\tilde{\tilde{d}}_{\tilde{r}}(\tilde{y})=\tilde{\tilde{d}}_{\tilde{r}}(\tilde{x}):=b \neq 0 .
$$

This assumption implies that, for every $k>1$, there is $n_{0}(k) \in \mathbb{N}$ such that the inclusion

$$
A\left(p, b r_{n}, k\right) \supseteq\left\{x_{n}, y_{n}\right\}
$$

holds for all integer numbers $n>n_{0}(k)$. Recall that

$$
A\left(p, b r_{n}, k\right)=\left\{x \in X: \frac{b r_{n}}{k} \leq d(x, p) \leq k b r_{n}\right\} .
$$


It follows from (2.21) that

$$
d\left(x_{n}, y_{n}\right) \leq \operatorname{diam}\left(A\left(p, b r_{n}, k\right)\right)
$$

if $n>n_{0}(k)$. Consequently,

$$
\frac{1}{b} \limsup _{n \rightarrow \infty} \frac{d\left(x_{n}, y_{n}\right)}{r_{n}} \leq \limsup _{n \rightarrow \infty} \frac{\operatorname{diam}\left(A\left(p, b r_{n}, k\right)\right)}{b r_{n}} .
$$

Letting $k \rightarrow 1$ on the right-hand side of the last inequality and using (2.4) we see that

$$
0 \leq \frac{1}{b} \limsup _{n \rightarrow \infty} \frac{d\left(x_{n}, y_{n}\right)}{r_{n}} \leq \lim _{k \rightarrow \infty}\left(\limsup _{n \rightarrow \infty} \frac{\operatorname{diam}\left(A\left(p, b r_{n}, k\right)\right)}{b r_{n}}\right)=0 .
$$

Hence

$$
\tilde{d}_{\tilde{r}}(\tilde{x}, \tilde{y})=\lim _{n \rightarrow \infty} \frac{d\left(x_{n}, y_{n}\right)}{r_{n}}=0 .
$$

It implies that $\tilde{x}$ and $\tilde{y}$ are mutually stable.

It still remains to show that there exists a finite limit

$$
\tilde{d}_{\tilde{r}}(\tilde{x}, \tilde{y})=\lim _{n \rightarrow \infty} \frac{d\left(x_{n}, y_{n}\right)}{r_{n}}
$$

if

$$
0 \neq \tilde{\tilde{d}}_{\tilde{r}}(\tilde{x}) \neq \tilde{\tilde{d}}_{\tilde{r}}(\tilde{y}) \neq 0 .
$$

For convenience we write

$$
q_{n}:=d\left(x_{n}, p\right), \quad t_{n}:=d\left(y_{n}, p\right)
$$

for $n \in \mathbb{N}$. Condition (2.23) implies that there are $\varepsilon>0$ and an integer number $n_{0}(\varepsilon)$ such that

$$
\max \left\{q_{n}, t_{n}\right\}>0 \quad \text { and } \quad\left|\frac{q_{n}}{t_{n}}-1\right| \geq \varepsilon
$$

for all $n \geq n_{0}(\varepsilon)$. It is clear that

$$
x_{n} \in S\left(p, q_{n}\right) \quad \text { and } \quad y_{n} \in S\left(p, t_{n}\right),
$$

where $S\left(p, q_{n}\right)$ and $S\left(p, t_{n}\right)$ are the spheres with the common center $p \in X$ and the radii $q_{n}$ and $t_{n}$, respectively. Consequently, we have the following inequalities

$$
\Delta\left(S\left(p, q_{n}\right), S\left(p, t_{n}\right)\right) \geq d\left(x_{n}, y_{n}\right) \geq \delta\left(S\left(p, q_{n}\right), S\left(p, t_{n}\right)\right),
$$


where the quantity $\delta\left(S\left(p, q_{n}\right), S\left(p, t_{n}\right)\right)$ is defined as in Lemma 2.2. Limit relations (2.8) and (2.6) imply

$$
\kappa_{0}=\lim _{n \rightarrow \infty} \frac{\Delta\left(S\left(p, q_{n}\right), S\left(p, t_{n}\right)\right)}{\left|q_{n}-t_{n}\right|}=\lim _{n \rightarrow \infty} \frac{\delta\left(S\left(p, q_{n}\right), S\left(p, t_{n}\right)\right)}{\left|q_{n}-t_{n}\right|} .
$$

Hence, using (2.25), we obtain

$$
\kappa_{0}=\lim _{n \rightarrow \infty} \frac{d\left(x_{n}, y_{n}\right)}{\left|q_{n}-t_{n}\right|}=\frac{1}{\left|\tilde{\tilde{d}}_{\tilde{r}}(\tilde{x})-\tilde{\tilde{d}}_{\tilde{r}}(\tilde{y})\right|} \lim _{n \rightarrow \infty} \frac{d\left(x_{n}, y_{n}\right)}{r_{n}},
$$

that implies

$$
\tilde{d}_{\tilde{r}}(\tilde{x}, \tilde{y})=\lim _{n \rightarrow \infty} \frac{d\left(x_{n}, y_{n}\right)}{r_{n}}=\kappa_{0}\left|\tilde{\tilde{d}}_{\tilde{r}}(\tilde{x})-\tilde{\tilde{d}}_{\tilde{r}}(\tilde{y})\right| .
$$

Thus $\tilde{x}$ and $\tilde{y}$ are mutually stable.

It can be proved that conditions (i) and (ii) from Theorem 2.1 are mutually independent in the sense that none of them follows another.

We conclude this section by simple corollary of statement $(i v)$ of Lemma 2.1.

Proposition 2.2. Let $(X, d)$ be a metric space and let $Y$ be an unbounded subspace $X$. Then $(X, d) \in \mathfrak{A}$ implies $(Y, d) \in \mathfrak{A}$.

\section{Uniqueness and logarithmic spirals}

For simplicity we will consider only logarithmic spirals having the pole at 0 . The polar equation of these spirals is

$$
\rho=k b^{\varphi},
$$

where $k$ and $b$ are constants, $k \in(0, \infty)$ and $b \in(0,1) \cup(1, \infty)$. The rotation of the polar axis on the angle $\varphi_{1}=-\frac{\ln k}{\ln b}$ transforms (3.1) to the form

$$
\rho=b^{\varphi} .
$$

Let us denote by $\mathbb{S}^{*}$ the set of all complex numbers lying on spiral (3.2) and let

$$
\mathbb{S}=\mathbb{S}^{*} \cup\{0\},
$$

i.e., $\mathbb{S}$ is the closure of $\mathbb{S}^{*}$ in the complex plane $\mathbb{C}$. In the following theorem we consider $\mathbb{S}$ as a metric space with the usual metric $d(z, w)=|z-w|$.

Theorem 3.1. Each pretangent space to $(\mathbb{S}, d)$ at infinity is unique, tangent and isometric to $\mathbb{S}$. 
We shall need the following lemma. Denote by $\mathbb{C}^{*}$ the multiplicative group of all nonzero complex numbers.

Lemma 3.1. $\mathbb{S}^{*}$ is a subgroup of the group $\mathbb{C}^{*}$.

Proof. As is well known, a non-void subset $H$ of a group $G$ is a subgroup of $G$ if and only if

$$
h g \in H \quad \text { and } \quad h^{-1} \in H
$$

for all $h, g \in H$. (See, for example, [10, Chapter 1, §2].) Let $z$ be a point of $\mathbb{C}^{*}$. It is clear from (3.2) that $z \in \mathbb{S}^{*}$ if and only if

$$
z=|z| \exp \left(i \log _{b}|z|\right) .
$$

The last equality implies

$$
z^{-1}=|z|^{-1} \exp \left(-i \log _{b}|z|\right)=\left|z^{-1}\right| \exp \left(i \log _{b}\left|z^{-1}\right|\right) .
$$

Hence $z^{-1}$ belongs to $\mathbb{S}^{*}$ if $z \in \mathbb{S}^{*}$. Similarly we obtain $z w \in \mathbb{S}^{*}$ if $z \in \mathbb{S}^{*}$ and $w \in \mathbb{S}^{*}$.

The next useful lemma describes the isometries of metric identifications of pseudometric spaces.

Lemma 3.2. Let $\left(X, d_{X}\right)$ be a pseudometric space, $\left(Y, d_{Y}\right)$ a metric space, $(\Omega, \rho)$ a metric identification of $\left(X, d_{X}\right)$ and $\pi: X \rightarrow \Omega$ the corresponding natural projection. Then for every distance-preserving, surjective mapping $F: X \rightarrow Y$ there is a unique mapping $f: \Omega \rightarrow Y$ such that the diagram

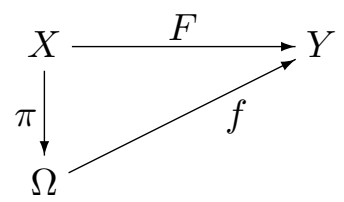

is commutative. The mapping $f$ is an isometry of metric spaces $(\Omega, \rho)$ and $\left(Y, d_{Y}\right)$.

Proof. Let us define a mapping $f$ by the rule: if $\alpha \in \Omega$, then we set

$$
f(\alpha):=F(x),
$$

where $x$ is an arbitrary point in $\pi^{-1}(\alpha)$. This definition is correct. Indeed, if $x_{1}, x_{2} \in \pi^{-1}(\alpha)$, then $d_{X}\left(x_{1}, x_{2}\right)=0$ because $\pi$ is natural projection. The equality $d_{X}\left(x_{1}, x_{2}\right)=0$ implies $d_{Y}\left(F\left(x_{1}\right), F\left(x_{2}\right)\right)=0$ because $F$ is distance-preserving. Since $d_{Y}$ is a metric, the last equality gives $F\left(x_{1}\right)=F\left(x_{2}\right)$. 
Rewriting (3.4) as $f(\pi(x))=F(x)$ we see that the diagram is commutative. The uniqueness of $f$ which satisfies the equality $F=f \circ \pi$ follows from the surjectivity of $\pi$. It still remains to prove that $F$ is an isometry.

Let $\alpha, \beta \in \Omega, x, y \in X$ and $\alpha=\pi(x), \beta=\pi(y)$. Then we have

$$
\begin{aligned}
d_{Y}(f(\alpha), f(\beta))=d_{Y}(f(\pi(x)), & f(\pi(y)))=d_{Y}(F(x), F(y)) \\
& =d_{X}(x, y)=\rho(\pi(x), \pi(y))=\rho(\alpha, \beta) .
\end{aligned}
$$

Thus $f$ is distance-preserving. Moreover $f$ is surjective because $F$ is surjective. Hence $f$ is an isometry as a distance-preserving, surjective mapping between metric spaces.

Proof of Theorem 3.1. We shall first prove $(\mathbb{S}, d) \in \mathfrak{U}$. To this end it is sufficient to show that conditions $(i)$ and (ii) from Theorem 2.1 are satisfied with $(X, d)=(\mathbb{S}, d)$.

We start from the verification of condition $(i)$.

Condition $(i)$. Let $z_{1}$ and $z_{2}$ be some points of the annulus

$$
A(0, r, k)=\left\{z \in \mathbb{S}: \frac{r}{k} \leq|z| \leq k r\right\},
$$

where $k \in(1, \infty), r \in(0, \infty)$. Let us denote by $l\left(z_{1}, z_{2}\right)$ the length of the arc of spiral (3.2) joining the points $z_{1}, z_{2}$. If the polar coordinates of $z_{1}$ and $z_{2}$ are $\left(\rho_{1}, \varphi_{1}\right)$ and $\left(\rho_{2}, \varphi_{2}\right)$ respectively, then we obtain the famous formula

$$
\begin{gathered}
l\left(z_{1}, z_{2}\right)=\left|\int_{\varphi_{1}}^{\varphi_{2}} \sqrt{\rho^{2}+\rho^{\prime 2}} d \varphi\right|=\frac{\sqrt{1+\ln ^{2} b}}{|\ln b|}\left|b^{\varphi_{2}}-b^{\varphi_{1}}\right| \\
=\frac{\sqrt{1+\ln ^{2} b}}{|\ln b|}\left|\rho_{2}-\rho_{1}\right| .
\end{gathered}
$$

It implies that

$$
\begin{aligned}
\operatorname{diam} A(0, r, k) \leq & \sup \left\{l\left(z_{1}, z_{2}\right): z_{1}, z_{2} \in A(0, r, k)\right\} \\
& =\frac{\sqrt{1+\ln ^{2} b}}{|\ln b|}\left|r k-r \frac{1}{k}\right|=\frac{r \sqrt{1+\ln ^{2} b}}{|\ln b|}\left(k-\frac{1}{k}\right) .
\end{aligned}
$$

Consequently,

$$
\lim _{k \rightarrow 1} \limsup _{r \rightarrow 0} \frac{\operatorname{diam}(A(0, r, k))}{r} \leq \lim _{k \rightarrow 1} \frac{\sqrt{1+\ln ^{2} b}}{|\ln b|}\left(k-\frac{1}{k}\right)=0,
$$

i.e., (2.4) holds and condition $(i)$ is satisfied. 
Condition (ii). Let $\varepsilon \in(0,1)$ and let $\left(\left(q_{n}, t_{n}\right)\right)_{n \in \mathbb{N}}$ be a sequence of points of $S_{\varepsilon}^{2}$ such that

$$
\lim _{n \rightarrow \infty} q_{n}=\lim _{n \rightarrow \infty} t_{n}=\infty
$$

and there is

$$
\lim _{n \rightarrow \infty} \frac{q_{n}}{t_{n}}=c_{0} \in[0, \infty]
$$

We must show that there exists a finite limit

$$
\lim _{n \rightarrow \infty} \frac{\Delta\left(S\left(0, q_{n}\right), S\left(0, t_{n}\right)\right)}{\left|q_{n}-t_{n}\right|}=\kappa_{0} .
$$

It follows from the definition of the set $S_{\varepsilon}^{2}$ that $q_{n}, t_{n} \in(0, \infty)$ for all $n \in \mathbb{N}$. Consequently, we can find $\theta_{n}, \tau_{n} \in(-\infty,+\infty)$ such that

$$
q_{n} e^{i \tau_{n}} \in \mathbb{S}^{*} \text { and } t_{n} e^{i \theta_{n}} \in \mathbb{S}^{*}
$$

for every $n \in \mathbb{N}$. Since the spheres $S\left(0, q_{n}\right)$ and $S\left(0, t_{n}\right)$ are single-point and $q_{n} e^{i \tau_{n}} \in S\left(0, q_{n}\right)$ and $t_{n} e^{i \theta_{n}} \in S\left(0, t_{n}\right)$, we have

$$
\frac{\Delta\left(S\left(0, t_{n}\right), S\left(0, q_{n}\right)\right)}{\left|t_{n}-q_{n}\right|}=\frac{\left|t_{n} e^{i \theta_{n}}-q_{n} e^{i \tau_{n}}\right|}{\left|t_{n}-q_{n}\right|}
$$

with

$$
\tau_{n}=\log _{b} q_{n}, \theta_{n}=\log _{b} t_{n},
$$

(see formula (3.3)). Consider firstly equation (3.7) with $c_{0}=0$ or $c_{0}=\infty$. If $c_{0}=0$, then we have

$$
\kappa_{0}=\lim _{n \rightarrow \infty} \frac{\left|t_{n} e^{i \theta_{n}}-q_{n} e^{i \tau_{n}}\right|}{\left|t_{n}-q_{n}\right|}=\lim _{n \rightarrow \infty} \frac{\left|1-\frac{q_{n}}{t_{n}} e^{i\left(\tau_{n}-\theta_{n}\right)}\right|}{\left|1-\frac{q_{n}}{t_{n}}\right|}=\frac{\left|1-c_{0}\right|}{\left|1-c_{0}\right|}=1 .
$$

Similar computations yield $\kappa_{0}=1$ for $c_{0}=\infty$. Suppose now $c_{0} \in(0, \infty)$. Using (3.7) and (3.10) we obtain

$$
\lim _{n \rightarrow \infty}\left(\tau_{n}-\theta_{n}\right)=\lim _{n \rightarrow \infty}\left(\log _{b} q_{n}-\log _{b} t_{n}\right)=\lim _{n \rightarrow \infty} \log _{b} \frac{q_{n}}{t_{n}}=\log _{b} c_{0} .
$$

Moreover, we have

$$
c_{0} \neq 1
$$

because $\left(\left(q_{n}, t_{n}\right)\right)_{n \in \mathbb{N}} \subset S_{\varepsilon}^{2}$. Applying (3.12) in (3.11) we obtain

$$
\lim _{n \rightarrow \infty} \frac{\left|t_{n} e^{i \theta_{n}}-q_{n} e^{i \tau_{n}}\right|}{\left|t_{n}-q_{n}\right|}=\frac{\left|1-c_{0} \exp \left(i \log _{b} c_{0}\right)\right|}{\left|1-c_{0}\right|} .
$$


Note that the right side in this equality is finite and well defined by virtue of (3.13).

Thus conditions $(i)$ and (ii) from Theorem 2.1 are satisfied, so that this theorem implies the desirable uniqueness of pretangent spaces. It still remains to prove that all pretangent spaces are tangent and isometric to $\mathbb{S}$.

Let $\tilde{r}=\left(r_{n}\right)_{n \in \mathbb{N}}$ be a scaling sequence and let $\tilde{\mathbb{S}}_{\infty, \tilde{r}}$ be the corresponding maximal self-stable set. For every $\tilde{x}=\left(x_{n}\right)_{n \in \mathbb{N}} \in \tilde{\mathbb{S}}_{\infty, \tilde{r}}$ there is a unique $x^{*} \in \mathbb{S}$ such that

$$
\left|x^{*}\right|=\lim _{n \rightarrow \infty} \frac{d\left(x_{n}, 0\right)}{r_{n}}=\tilde{\tilde{d}}_{\tilde{r}}(\tilde{x}) .
$$

We claim that the mapping

$$
F: \tilde{\mathbb{S}}_{\infty, \tilde{r}} \rightarrow \mathbb{S}, \quad F(\tilde{x})=x^{*}
$$

is surjective and distance-preserving in the sense that the equality

$$
\tilde{d}_{\tilde{r}}(\tilde{x}, \tilde{y})=\left|x^{*}-y^{*}\right|
$$

holds for all $\tilde{x}, \tilde{y} \in \tilde{\mathbb{S}}_{\infty, \tilde{r}}$.

Surjectivity. We have already verified that $(\mathbb{S}, d) \in \mathfrak{U}$. Hence, by Lemma 2.1 , it is sufficient to prove that for every $a \in \mathbb{S}$ there is $\tilde{x} \in \tilde{\mathbb{S}}_{\infty, \tilde{r}}$ such that $\left|x^{*}\right|=|a|$. If $|a|=0$, then the equality $\left|x^{*}\right|=|a|$ is evident for $\tilde{x} \in \tilde{\mathbb{S}}_{\infty, \tilde{r}}^{0}$. Assume that $|a|>0$. For every $n \in \mathbb{N}$ define a complex number $z_{n}^{0}$ as

$$
z_{n}^{0}:=\left|\frac{1}{r_{n}}\right| \exp \left(i \log \left|\frac{1}{r_{n}}\right|\right)
$$

(see formula (3.3)). The points $z_{n}^{0}$ and $x^{*}$ belong to $\mathbb{S}^{*}$. By Lemma 3.1 the set $\mathbb{S}^{*}$ is a subgroup of $\mathbb{C}^{*}$. Since the equation $c y=b$ is solvable in every group with given $b$ and $c$ belonging to this group, there is $x_{n} \in \mathbb{S}^{*}$ such that

$$
z_{n}^{0} x_{n}=a .
$$

This equality and (3.17) imply

$$
\frac{d\left(x_{n}, 0\right)}{r_{n}}=\left|\frac{x_{n}}{z_{n}^{0}}\right|=|a|
$$

for every $n \in \mathbb{N}$. Thus the equality $|a|=\left|x^{*}\right|$ holds for $\tilde{x}:=\left(x_{n}\right)_{n \in \mathbb{N}}$ if every $x_{n}$ fulfills (3.18).

Preservation of distances. Let $\tilde{x}$ and $\tilde{y} \in \tilde{\mathbb{S}}_{\infty, \tilde{r}}$. If $x^{*}=0$ or $y^{*}=0$, then equality (3.16) follows simply from (3.14). Assume $x^{*} \neq 0 \neq y^{*}$. This assumption implies $x^{*} \in \mathbb{S}^{*}, y^{*} \in \mathbb{S}^{*}$ and

$$
x_{n} \in \mathbb{S}^{*}, \quad y_{n} \in \mathbb{S}^{*}
$$


for all sufficiently large $n$. These membership relations give, in particular, the equalities

$$
x^{*}=\left|x^{*}\right| \exp \left(i \log _{b}\left|x^{*}\right|\right) \quad \text { and } \quad x_{n}=\left|x_{n}\right| \exp \left(i \log _{b}\left|x_{n}\right|\right),
$$

see (3.3). Moreover, we can rewrite (3.17) as

$$
z_{n}^{0}=\left|z_{n}^{0}\right| \exp \left(i \log _{b}\left|z_{n}^{0}\right|\right) .
$$

Using it, (3.19) and (3.14) we obtain

$$
\lim _{n \rightarrow \infty}\left|z_{n}^{0}\right|\left|x_{n}\right|=\left|x^{*}\right|
$$

Consequently,

$$
\lim _{n \rightarrow \infty} \exp \left(i \log _{b}\left(\left|z_{n}^{0}\right|\left|x_{n}\right|\right)\right)=\exp \left(i \log _{b}\left|x^{*}\right|\right) .
$$

Relations (3.19)-(3.21) give the equality

$$
\lim _{n \rightarrow \infty} z_{n}^{0} x_{n}=x^{*}
$$

Similarly, we have $\lim _{n \rightarrow \infty} z_{n}^{0} y_{n}=y^{*}$. The last two limit relations imply

$$
\left|x^{*}-y^{*}\right|=\left|\lim _{n \rightarrow \infty}\left(r_{n}^{0} x_{n}-r_{n}^{0} y_{n}\right)\right|=\lim _{n \rightarrow \infty} \frac{\left|x_{n}-y_{n}\right|}{r_{n}},
$$

i.e., (3.16) holds.

We can now easily show that all pretangent spaces $\Omega_{\infty, \tilde{r}}^{\mathbb{S}}$ are isometric to $\mathbb{S}$ and tangent. Indeed, as has been shown above the mapping $F: \tilde{\mathbb{S}}_{\infty, \tilde{r}} \rightarrow \mathbb{S}$ is distance-preserving and onto. Hence, by Lemma 3.2, there is an isometry $f: \Omega_{\infty, \tilde{r}}^{\mathbb{S}} \rightarrow \mathbb{S}$. All $\Omega_{\infty, \tilde{r}}^{\mathbb{S}}$ are tangent if and only if all mappings $\varphi_{\tilde{r}^{\prime}}: \tilde{\mathbb{S}}_{\infty, \tilde{r}} \rightarrow \widetilde{\mathbb{S}}_{\infty, \tilde{r}^{\prime}}$ are surjective (see diagram (1.5) and Remark 1.2). Let $A$ be an infinite subset of $\mathbb{N}, \tilde{r}^{\prime}=\left(r_{n}\right)_{n \in A}$ be the subsequence of $\tilde{r}=\left(r_{n}\right)_{n \in \mathbb{N}}$ and let $\tilde{y}=\left(y_{n}\right)_{n \in A} \in \tilde{\mathbb{S}}_{\infty, \tilde{r}^{\prime}}$. Suppose that $\tilde{\tilde{d}}_{\tilde{r}^{\prime}}(\tilde{y})>0$ (the case $\tilde{\tilde{d}}_{\tilde{r}^{\prime}}(\tilde{y})=0$ is more simple and can be considered similarly). Let us define $x_{n}$ as

$$
x_{n}:= \begin{cases}y_{n} & \text { if } n \in A \\ \frac{y}{z_{n}^{0}} & \text { if } n \in \mathbb{N} \backslash A,\end{cases}
$$

where $z_{n}^{0}$ is defined by (3.17) and $y$ is a point of $\mathbb{S}^{*}$ such that $|y|=\tilde{\tilde{d}}_{\tilde{r}^{\prime}}(\tilde{y})$. It follows from Lemma 3.1 that $\tilde{x}=\left(x_{n}\right)_{n \in \mathbb{N}} \subset \mathbb{S}$. Moreover, (3.17) and (3.23) imply

$$
\lim _{n \rightarrow \infty} \frac{d\left(x_{n}, 0\right)}{r_{n}}=|y|<\infty
$$


Consequently, $\tilde{x} \in S e q(\mathbb{S}, \tilde{r})$ holds. It is also clear that $\tilde{x}^{\prime}:=\left(x_{n}\right)_{n \in A}=\tilde{y}$. By Lemma 2.1, $(\mathbb{S}, d) \in \mathfrak{U}$ implies $\tilde{\mathbb{S}}_{\infty, \tilde{r}}=\operatorname{Seq}(\mathbb{S}, \tilde{r})$. Hence each $\varphi_{\tilde{r}^{\prime}}$ is a surjective mapping.

Remark 3.1. Lemma 3.1 is closely related to the contemporary definition of logarithmic spirals (see, for example, [3, 9.6.9.1]). In the proof of Theorem 3.1 we have used formula (3.5) but we really need only in the following remarkable fact discovered by Rene Descartes: The length measured along the logarithmic spiral from the pole $O$ to the point $P$ of the spiral is proportional to the radius vector $O P$.

The logarithmic spirals and the set $\mathbb{R}_{+}^{*}:=\mathbb{R}^{+} \backslash\{0\}$ of all strictly positive real numbers have some common properties: They are unbounded subgroups of the multiplicative group $\mathbb{C}^{*}$ and have unique pretangent spaces at infinity. The following theorem shows that the logarithmic spirals and $\mathbb{R}_{+}^{*}$ are exhausted all maximal subgroups of $\mathbb{C}^{*}$ having these properties.

In what follows we denote by $\mathbb{S}^{*}(b)$ the set of complex numbers lying on spiral (3.2).

Theorem 3.2. Let $\Gamma^{*}$ be an unbounded subgroup of the multiplicative group $\mathbb{C}^{*}$. The following two statements are equivalent.

(i) $\Gamma^{*} \subseteq \mathbb{R}_{+}^{*}$ or there is $b \in(0,1) \cup(1, \infty)$ such that $\Gamma^{*} \subseteq \mathbb{S}^{*}(b)$.

(ii) $\Gamma^{*} \in \mathfrak{U}$.

Proof. First of all we claim that the theorem is true for all unbounded $\Gamma^{*}$ if and only if it is true for all closed (in $\mathbb{C}^{*}$ ), unbounded $\Gamma^{*}$. Let $\bar{\Gamma}^{*}$ be the closure of $\Gamma^{*}$ in $\mathbb{C}^{*}$. For convenience we denote by $\overline{(i)}$ and $\overline{(i i)}$ the statements obtained by the substitution of $\bar{\Gamma}^{*}$ for $\Gamma^{*}$ in $(i)$ and, respectively, in $(i i)$. Since $\mathbb{R}_{+}^{*}$ and $\mathbb{S}^{*}(b)$ are closed subsets of $\mathbb{C}^{*}$ we have the equivalences

$$
\left(\bar{\Gamma}^{*} \subseteq \mathbb{R}_{+}^{*}\right) \Leftrightarrow\left(\Gamma^{*} \subseteq \mathbb{R}_{+}^{*}\right) \quad \text { and } \quad\left(\bar{\Gamma}^{*} \subseteq \mathbb{S}^{*}(b)\right) \Leftrightarrow\left(\Gamma^{*} \subseteq \mathbb{S}^{*}(b)\right) .
$$

Thus $\overline{(i)} \Leftrightarrow(i)$ holds. Using Lemma 3.2 we see that $(i i) \Leftrightarrow \overline{(i i)}$. Moreover, since the closure of a subgroup is a subgroup [10, p. 102], $\bar{\Gamma}^{*}$ is a closed subgroup of $\mathbb{C}^{*}$.

Thus we may assume, without loss of generality, that $\Gamma^{*}$ is a closed subgroup of $\mathbb{C}^{*}$.

(i) $\Rightarrow$ (ii) This implication follows from Proposition 2.2, Theorem 3.1 and Proposition 2.1. Indeed, Proposition 2.1 claims, in particular, that $\mathbb{R}^{+}$has a unique pretangent space at infinity and, by Theorem 3.1 , the 
same property is true for $\mathbb{S}^{*}(b)$. Finally, the property "to have a unique pretangent space" is hereditary by Proposition 2.2.

$($ ii $) \Rightarrow(i)$ Suppose that $\Gamma^{*}$ is an unbounded, closed subgroup of $\mathbb{C}^{*}$ and $(i i)$ holds. Since $\Gamma^{*}$ is unbounded, there exists a sequence $\left(p_{i}\right)_{i \in \mathbb{N}} \subset$ $\Gamma^{*}$ with

$$
\lim _{i \rightarrow \infty}\left|p_{i}\right|=\infty .
$$

Let $z_{1}, z_{2} \in \Gamma^{*}$ and let $\left|z_{1}\right|=\left|z_{2}\right|$ hold. Write $r_{i}=\frac{\left|z_{1}\right|}{\left|p_{i}\right|}, i \in \mathbb{N}$. Note that the points $\frac{z_{1}}{p_{i}}$ and $\frac{z_{2}}{p_{i}}$ belong to $\Gamma^{*}$ because $\Gamma^{*}$ is a subgroup of $\mathbb{C}^{*}$. It is clear that

$$
\frac{z_{j}}{p_{i}} \in A\left(0, r_{i}, k\right)=\left\{z \in \Gamma^{*}: \frac{r_{i}}{k} \leq|z| \leq k r_{i}\right\}, \quad j=1,2,
$$

for all $k \in[1, \infty)$ and $i \in \mathbb{N}$. Statement (ii) of the present theorem implies (2.4). Consequently, by Theorem 2.1, we have

$$
0=\lim _{k \rightarrow 1} \limsup _{i \rightarrow \infty} \frac{\operatorname{diam}\left(A\left(0, r_{i}, k\right)\right)}{r_{i}} \geq \frac{\left|\frac{z_{1}}{p_{i}}-\frac{z_{2}}{p_{i}}\right|}{\left|\frac{z_{1}}{p_{i}}\right|}=\frac{\left|z_{1}-z_{2}\right|}{\left|z_{1}\right|},
$$

i.e., the implication

$$
\left(\left|z_{1}\right|=\left|z_{2}\right|\right) \Rightarrow\left(z_{1}=z_{2}\right)
$$

is valid for all $z_{1}, z_{2} \in \Gamma^{*}$.

Let us consider the continuous homomorphism $\Phi$ from the group $\Gamma^{*}$ to the group $\mathbb{R}_{+}^{*}$ defined as

$$
\Phi(z)=|z|, \quad z \in \Gamma^{*} .
$$

Then $\Phi$ is closed. Consequently, $\Phi\left(\Gamma^{*}\right)$ is a closed subgroup of $\mathbb{R}_{+}^{*}$. Using the well-known classification of the closed subgroups of the additive group for real numbers (see, for example, [7, Chapter V, 11,1$]$ ), we obtain the following three possibilities:

(i) $\Phi\left(\Gamma^{*}\right)=\{1\}$;

(ii) $\Phi\left(\Gamma^{*}\right)=\mathbb{R}_{+}^{*}$;

(iii) There is $g \in(1, \infty)$ such that $\Phi\left(\Gamma^{*}\right)=\left\{g^{n}: n \in \mathbb{Z}\right\}$, where $\mathbb{Z}$ is the set of integers.

Since $\Gamma^{*}$ is unbounded, the case $\left(i_{1}\right)$ is impossible. Implication (3.25) shows that the homomorphism $\Phi: \Gamma^{*} \rightarrow \mathbb{R}_{+}^{*}$ is one-to-one. Consequently, for the case $\left(i_{3}\right)$, the group $\Gamma^{*}$ is cyclic with the generator $z=\Phi^{-1}(g)$. 
Writing $z$ in the trigonometric form $z=|z| e^{i \varphi}=g e^{i \varphi}, \varphi \in[0,2 \pi)$, we see that either $\Gamma^{*} \subseteq \mathbb{R}_{+}^{*}$ for $\varphi=0$ or, for $\varphi \in(0,2 \pi), \Gamma^{*}$ lies on the logarithmic spiral

$$
\mathbb{S}^{*}(b)=\left\{t \exp \left(i \log _{b} t\right): t \in \mathbb{R}_{+}^{*}\right\}
$$

with $b=\exp \left(\frac{\ln g}{\varphi}\right)$, where $\ln g$ is the natural logarithm of $g$.

Suppose now that we have $\Phi\left(\Gamma^{*}\right)=\mathbb{R}_{+}^{*}$. Then $\Phi$ is an isomorphism of the groups $\Gamma^{*}$ and $\mathbb{R}_{+}^{*}$ and, simultaneously, $\Phi$ is a homeomorphism as a continuous, closed bijection of the topological spaces $\Gamma^{*}$ and $\mathbb{R}_{+}^{*}$. Write $\Phi^{-1}$ for the inverse mapping of $\Phi$ and

$$
\mathbb{T}:=\{z \in \mathbb{C}:|z|=1\} .
$$

Let $\Psi: \mathbb{C}^{*} \rightarrow \mathbb{T}$ be the standard homomorphism, $\Psi(z)=\frac{z}{|z|}$ and let in: $\Gamma^{*} \rightarrow \mathbb{C}^{*}$ be the inclusion, $i n(z)=z$. Then the mapping

$$
\mathbb{R} \stackrel{\exp }{\longrightarrow} \mathbb{R}_{+}^{*} \stackrel{\Phi^{-1}}{\longrightarrow} \Gamma^{*} \stackrel{i n}{\longrightarrow} \mathbb{C}^{*} \stackrel{\Psi}{\longrightarrow} \mathbb{T}
$$

is a character (a continuous homomorphism) of the additive group $\mathbb{R}$. Denote this character by $\varkappa$. Then there is $\nu \in \mathbb{R}$ such that

$$
\varkappa(t)=\exp (i \nu t)
$$

holds for all $t \in \mathbb{R}$ (see [10, p. 271]). Since $\Phi^{-1}$ is bijective, from (3.26) and (3.27) we obtain that

$$
\varkappa(t)=\frac{\Phi^{-1}(\exp t)}{\exp t}=\exp (i \nu t), \quad t \in \mathbb{R}
$$

i.e.,

$$
\begin{gathered}
\frac{\Phi^{-1}(|z|)}{|z|}=\exp (i \nu \ln |z|), \\
z=|z| \exp (i \nu \ln |z|)
\end{gathered}
$$

hold for every $z \in \Gamma^{*}$. The last equality implies $\Gamma^{*} \subseteq \mathbb{R}_{+}^{*}$ if $\nu=0$. For $\nu \neq 0$ we can rewrite $(3.28)$ as

$$
z=|z| \exp \left(i \log _{b}|z|\right),
$$

where $b=\exp \left(\frac{1}{\nu}\right)$. Hence $\Gamma^{*}$ is a logarithmic spiral with the pole at 0 if $\nu \neq 0$. 


\section{Uniqueness for subsets of $\mathbb{R}$}

In this section we specify the uniqueness conditions, presented by Theorem 2.1 for the general, unbounded metric spaces $X$, to the case $X \subseteq \mathbb{R}$.

Lemma 4.1. Let $X \subseteq \mathbb{R}$ be unbounded and let $d(x, y)=|x-y|$ for all $x, y \in X$. Condition ( $i$ ) from Theorem 2.1 does not hold if and only if there exist some sequences $\tilde{x}, \tilde{y} \subset X$ such that

$$
\lim _{n \rightarrow \infty} x_{n}=\lim _{n \rightarrow \infty} y_{n}=\infty \quad \text { and } \quad x_{n} \in(-\infty, 0), y_{n} \in(0,+\infty)
$$

for every $n \in \mathbb{N}$ and

$$
\lim _{n \rightarrow \infty} \frac{x_{n}}{y_{n}}=-1 .
$$

Proof. Without loss of generality we can suppose that $0 \in X$.

Let $\tilde{x}$ and $\tilde{y}$ satisfy relations (4.1) and (4.2). For every $n \in \mathbb{N}$, write

$$
r_{n}:=\sqrt{\left|x_{n}\right|\left|y_{n}\right|} \quad \text { and } \quad k_{n}:=\max \left\{\left|\frac{x_{n}}{y_{n}}\right|^{\frac{1}{2}},\left|\frac{y_{n}}{x_{n}}\right|^{\frac{1}{2}}\right\} .
$$

It is easy to see that

$$
\lim _{n \rightarrow \infty} r_{n}=\infty \text { and } \quad \lim _{n \rightarrow \infty} k_{n}=1,
$$

and $r_{n}>0$, and $k_{n} \geq 1$, and

$$
x_{n}, y_{n} \in A\left(0, r_{n}, k_{n}\right)=\left\{x \in X: \frac{r_{n}}{k_{n}} \leq|x| \leq r_{n} k_{n}\right\}
$$

for every $n \in \mathbb{N}$. Now, using the inequality of arithmetic and geometric means, we obtain

$$
\frac{\operatorname{diam}\left(A\left(0, r_{n}, k_{n}\right)\right)}{r_{n}} \geq \frac{\left|y_{n}-x_{n}\right|}{r_{n}}=\frac{\left|y_{n}\right|+\left|x_{n}\right|}{r_{n}}=\sqrt{\frac{\left|y_{n}\right|}{\left|x_{n}\right|}}+\sqrt{\frac{\left|x_{n}\right|}{\left|y_{n}\right|}} \geq 2 .
$$

Hence the inequality

$$
\lim _{k \rightarrow 1} \limsup _{r \rightarrow \infty} \frac{A(0, r, k)}{r} \geq 2
$$

holds. Thus condition $(i)$ of Theorem 2.1 does not hold.

Conversely, if condition $(i)$ does not hold, then we have

$$
\lim _{k \rightarrow 1} \limsup _{r \rightarrow \infty} \frac{\operatorname{diam}(A(0, r, k))}{r}>0 .
$$


We must find sequences $\tilde{x}, \tilde{y} \subset X$ which satisfy relations (4.1) and (4.2). Inequality (4.3) implies that there are a constant $c>0$ and some sequences $\left(r_{n}\right)_{n \in \mathbb{N}} \subset(0, \infty)$ and $\left(k_{n}\right)_{n \in \mathbb{N}} \subset[1, \infty)$ such that

$$
\lim _{n \rightarrow \infty} r_{n}=\infty \quad \text { and } \quad \lim _{n \rightarrow \infty} k_{n}=1
$$

and

$$
\frac{\operatorname{diam} A\left(0, r_{n}, k_{n}\right)}{r_{n}}>c
$$

for every $n \in \mathbb{N}$. Let us consider the closed intervals

$$
I_{n}^{+}:=\left[\frac{r_{n}}{k_{n}}, r_{n} k_{n}\right], \quad I_{n}^{-}:=\left[-r_{n} k_{n},-\frac{r_{n}}{k_{n}}\right] .
$$

It is clear that

$$
A\left(0, r_{n}, k_{n}\right) \subseteq I_{n}^{+} \cup I_{n}^{-} .
$$

Inequality (4.4) implies, for every $n \in \mathbb{N}$, there are $x_{n}, y_{n} \in A\left(0, r_{n}, k_{n}\right)$ such that $x_{n}<y_{n}$ and

$$
\frac{\left|x_{n}-y_{n}\right|}{r_{n}}>c .
$$

If $x_{n}, y_{n} \in I_{n}^{+}$or $x_{n}, y_{n} \in I_{n}^{-}$, then

$$
\frac{\left|x_{n}-y_{n}\right|}{r_{n}} \leq k_{n}-\frac{1}{k_{n}} .
$$

Since $\lim _{n \rightarrow \infty} k_{n}=1$, inequality (4.6) contradicts (4.5) for sufficiently large $n$. Hence

$$
x_{n} \in I_{n}^{-} \quad \text { and } \quad y_{n} \in I_{n}^{+}
$$

if $n$ is large enough. Relations (4.7) and $\lim _{n \rightarrow \infty} k_{n}=1$ imply (4.2). The rest of desirable properties of $\tilde{x}=\left(x_{n}\right)_{n \in \mathbb{N}}$ and $\tilde{y}=\left(y_{n}\right)_{n \in \mathbb{N}}$ are evident by construction.

Let $X \subseteq \mathbb{R}$ and $p \in \mathbb{R}$. Recall that $X$ is locally symmetric at a point $p \in \mathbb{R}$ if there is $\varepsilon>0$ such that

$$
(x+p \in X) \Leftrightarrow(-x+p \in X)
$$

holds for every $x \in X \cap(p-\varepsilon, p+\varepsilon)$. (See, for example, [12, p. 225].)

We shall say a set $X \subseteq \mathbb{R}$ is asymmetric at infinity with respect to a point $p \in \mathbb{R}$ if there is $\varepsilon>0$ such that

$$
(x+p \in X) \Rightarrow(-x+p \notin X)
$$

for each $x \in X \cap((-\infty, p-\varepsilon) \cup(p+\varepsilon, \infty))$. 
Corollary 4.1. Let $X \subseteq \mathbb{R}$ be unbounded. If condition ( $i)$ from Theorem 2.1 holds, then $X$ is asymmetric at infinity with respect to every point $p \in \mathbb{R}$.

Example 4.1 (Asymmetric at Infinity Subset of $\mathbb{R}$ ). Let $\left(r_{n}\right), n \in \mathbb{N} \cup$ $\{0\}$, be a strictly convex sequence with $r_{1}>r_{0}=0$ and

$$
\lim _{n \rightarrow \infty}\left(r_{n+1}-r_{n}\right)=\infty
$$

Recall that the strict convexity of $\left(r_{n}\right)$ means that the second order differences

$$
\Delta^{2} r_{n}=r_{n+2}-2 r_{n+1}-r_{n}
$$

are strictly positive for every $n \geq 0$. It implies

$$
r_{n+2}-r_{n+1}>r_{n+1}-r_{n}>\ldots>r_{1}-r_{0}=r_{1}>0 .
$$

Write

$$
X^{+}:=\bigcup_{n=1}^{\infty}\left[r_{4 n}, r_{4 n+1}\right], \quad X^{-}:=\bigcup_{n=1}^{\infty}\left[-r_{4 n-1},-r_{4 n-2}\right]
$$

and define

$$
X:=X^{+} \cup X^{-} .
$$

It is clear that $X$ is an unbounded subset of $\mathbb{R}$. We claim that $X$ is asymmetric at infinity with respect all points $p \in \mathbb{R}$.

Let $p \in \mathbb{R}$ be given. We must show that $-x+p \notin X$ if $x+p \in X$ and $|x|$ is large enough. Using (4.9) and (4.10) we can find $n_{0} \in \mathbb{N}$ such that

$$
2|p|<r_{n_{0}}-r_{n_{0}-1} \text {. }
$$

If $x+p \in X$, then there is $m \in \mathbb{N} \cup\{0\}$ such that either

$$
x+p>0 \quad \text { and } \quad x+p \in\left[r_{4 m}, r_{4 m+1}\right]
$$

or

$$
x+p<0 \quad \text { and } \quad x+p \in\left[-r_{4 m-1},-r_{4 m-2}\right] .
$$

Suppose (4.12) holds (case (4.13) is similar). For sufficiently large $x$ we obtain the inequality $4 m>n_{0}$. It follows from (4.12) that

$$
-x+p \in\left[-r_{4 m+1}+2 p,-r_{4 m}+2 p\right] \subseteq\left[-r_{4 m+1}-2|p|,-r_{4 m}+2|p|\right] .
$$

Now, using the inequality $4 m>n_{0},(4.12),(4.10)$ we obtain

$$
\begin{gathered}
-x+p \in\left[-r_{4 m+1}-\left|r_{n_{0}}-r_{n_{0}-1}\right|,-r_{4 m}+\left|r_{n_{0}}-r_{n_{0}-1}\right|\right] \\
\subset\left(-r_{4 m+2},-r_{4 m-1}\right) .
\end{gathered}
$$

It follows from the definition of the set $X$, that the intersection of $X$ with the interval $\left(-r_{4 m+2},-r_{4 m-1}\right)$ is empty. Thus we have $-x+p \notin X$. 
Consider now the "real-valued" variant of condition (ii) from Theorem 2.1.

Let $X \subseteq \mathbb{R}$ be asymmetric at infinity with respect to $p \in X$. Write

$$
\begin{aligned}
& X_{p}^{+}:=\{x \in X: x+p \in X, x+p \geq 0\}, \\
& X_{p}^{-}:=\{x \in X: x+p \in X, x+p \leq 0\} .
\end{aligned}
$$

Define the subsets ${ }_{+1} R_{p, X}$ and ${ }_{-1} R_{p, X}$ of the set $\mathbb{R}^{+}$by the rules:

$$
{ }_{+1} R_{p, X}:=\left\{|x-p|: x \in X_{p}^{+}\right\} \quad \text { and } \quad{ }_{-1} R_{p, X}:=\left\{|x-p|: x \in X_{p}^{-}\right\} .
$$

Then we obtain

$$
S p(X)={ }_{+1} R_{p, X} \cup{ }_{-1} R_{p, X} \quad \text { and } \quad{ }_{+1} R_{p, X} \cap{ }_{-1} R_{p, X} \cap[\delta, \infty)=\varnothing,
$$

for sufficiently large $\delta>0$. Simple geometric considerations show that, for all $q, t \in S p(X) \cap[\delta, \infty)$, we have

$$
\Delta(S(p, q), S(p, t))= \begin{cases}|q-t| & \text { if }(q, t) \in\left({ }_{+1} R_{p, X}^{2}\right) \cup\left({ }_{-1} R_{p, X}^{2}\right), \\ |q+t| & \text { otherwise }\end{cases}
$$

where ${ }_{+1} R_{p, X}^{2}$ and ${ }_{-1} R_{p, X}^{2}$ are the Cartesian squares of ${ }_{+1} R_{p, X}$ and, respectively, of ${ }_{-1} R_{p, X}$ and

$$
\Delta(S(p, q), S(p, t))=\sup \{|x-y|: x \in S(p, q), y \in S(p, t)\} .
$$

For every $\delta>0$, let us introduce also the sets

$$
\begin{gathered}
{ }_{+} K_{\delta}:=\left\{\frac{q}{t}: q, t \in{ }_{+1} R_{p, X} \cap[\delta, \infty)\right\}, \\
-K_{\delta}:=\left\{\frac{q}{t}: q, t \in{ }_{-1} R_{p, X} \cap[\delta, \infty)\right\}, \\
{ }_{1} K_{\delta}:=\left\{\frac{q}{t}:(q, t) \in\left(S p^{2}(X) \cap[\delta, \infty)^{2}\right) \backslash\left({ }_{+1} R_{p, X}^{2} \cup{ }_{-1} R_{p, X}^{2}\right)\right\},
\end{gathered}
$$

where $[\delta, \infty)^{2}$ and $S p^{2}(X)$ are the Cartesian squares of the infinite interval $[\delta, \infty)$ and $S p(X)$.

Proposition 4.1. Let $X \subseteq \mathbb{R}$ be unbounded and asymmetric at infinity with respect to $p \in X$. Condition (ii) of Theorem 2.1 holds if and only if

$$
\bigcap_{\delta \in \mathbb{R}_{+}^{*}}\left(C l\left({ }_{+} K_{\delta} \cup{ }_{-} K_{\delta}\right) \cap C l\left({ }_{1} K_{\delta}\right)\right) \subseteq\{0,1, \infty\},
$$

where $\mathbb{R}_{+}^{*}=(0, \infty)$ and the closures are taken in $[0, \infty]$. 
Proof. Suppose inclusion (4.16) holds. Let $\varepsilon_{0}>0$ and let $\left(\left(q_{n}, t_{n}\right)\right)_{n \in \mathbb{N}} \subset$ $S_{\varepsilon_{0}}^{2}$ such that $\lim _{n \rightarrow \infty} q_{n}=\lim _{n \rightarrow \infty} t_{n}=\infty$ and

$$
\lim _{n \rightarrow \infty} \frac{q_{n}}{t_{n}}:=c_{0} \in[0, \infty] .
$$

It is necessary to show that there is a finite limit

$$
\lim _{n \rightarrow \infty} \frac{\Delta\left(S\left(p, q_{n}\right), S\left(p, t_{n}\right)\right)}{\left|q_{n}-t_{n}\right|}=\varkappa_{0} .
$$

We first note that (4.18) holds with $\varkappa_{0}=1$ if $c_{0}=0$ or $c_{0}=\infty$. Indeed, equality (4.14) implies the double estimation

$$
\frac{\left|q_{n}-t_{n}\right|}{\left|q_{n}-t_{n}\right|} \leq \frac{\Delta\left(S\left(p, q_{n}\right), S\left(p, t_{n}\right)\right)}{\left|q_{n}-t_{n}\right|} \leq \frac{\left|q_{n}+t_{n}\right|}{\left|q_{n}-t_{n}\right|}
$$

Letting $n$ to infinity and using (4.17) with $c_{0} \in\{0, \infty\}$ we obtain (4.18) with $\varkappa_{0}=1$. Let us consider now the case $0<c_{0}<\infty$. Define, for $\delta>0$,

$$
K_{\delta}:=\left\{\frac{q}{t}: q, t \in S p(X) \cap[\delta, \infty)\right\} .
$$

Then, using the standard inclusion from the theory of cluster sets (see, for example, $[8,1.1])$, we have

$$
c_{0} \in \bigcap_{\delta \in \mathbb{R}_{+}^{*}} C l\left(K_{\delta}\right) .
$$

Furthermore, it follows from (4.15) that

$$
K_{\delta}={ }_{1} K_{\delta} \cup\left({ }_{+} K_{\delta} \cup{ }_{-} K_{\delta}\right) .
$$

Thus $C l\left(K_{\delta}\right)=C l\left({ }_{1} K_{\delta}\right) \cup C l\left({ }_{+} K_{\delta} \cup{ }_{-} K_{\delta}\right)$. The last equality and the monotonicity: "if $\delta_{1} \geq \delta_{2}$, then

$$
C l\left({ }_{1} K_{\delta_{1}}\right) \subseteq C l\left({ }_{1} K_{\delta_{2}}\right) \quad \text { and } \quad C l\left({ }_{+} K_{\delta_{1}} \cup{ }_{-} K_{\delta_{1}}\right) \subseteq C l\left({ }_{+} K_{\delta_{2}} \cup{ }_{-} K_{\delta_{2}}\right), "
$$

imply the equality

$$
\bigcap_{\delta \in \mathbb{R}_{+}^{*}} C l\left(K_{\delta}\right)=\left(\bigcap_{\delta \in \mathbb{R}_{+}^{*}} C l\left({ }_{1} K_{\delta}\right)\right) \bigcup\left(\bigcap_{\delta \in \mathbb{R}_{+}^{*}} C l\left({ }_{+} K_{\delta} \cup{ }_{-} K_{\delta}\right)\right) .
$$

Hence we have

$$
c_{0} \in \bigcap_{\delta \in \mathbb{R}_{+}^{*}} C l\left({ }_{1} K_{\delta}\right)
$$


or

$$
c_{0} \in \bigcap_{\delta \in \mathbb{R}_{+}^{*}} C l\left({ }_{+} K_{\delta} \cup{ }_{-} K_{\delta}\right) .
$$

It follows directly form (4.16) that

$$
\left(\bigcap_{\delta \in \mathbb{R}_{+}^{*}} C l\left({ }_{1} K_{\delta}\right)\right) \bigcap\left(\bigcap_{\delta \in \mathbb{R}_{+}^{*}} C l\left({ }_{+} K_{\delta} \cup{ }_{-} K_{\delta}\right)\right) \subseteq\{0,1, \infty\} .
$$

The relation $\left(q_{n}, t_{n}\right) \in S_{\varepsilon_{0}}^{2}$ imply the inequality

$$
\left|\frac{q_{n}}{t_{n}}-1\right| \geq \varepsilon_{0},
$$

so that

$$
c_{0} \neq 1 \text {. }
$$

Since $c_{0} \notin\{0,1, \infty\},(4.21)$ and (4.22) imply that there is $n_{0} \in \mathbb{N}$ such that either

$$
\left(q_{n}, t_{n}\right) \in S p^{2}(X) \backslash\left({ }_{+1} R_{p, X}^{2} \cup{ }_{-1} R_{p, X}^{2}\right)
$$

for every $n \geq n_{0}$, or

$$
\left(q_{n}, t_{n}\right) \in{ }_{+1} R_{p, X}^{2} \cup{ }_{-1} R_{p, X}^{2}
$$

for every $n \geq n_{0}$. Now applying (4.14) we obtain

$$
\varkappa_{0}= \begin{cases}\frac{\left|1+c_{0}\right|}{\left|1-c_{0}\right|} & \text { if }(4.23) \text { holds } \\ 1 & \text { if }(4.24) \text { holds } .\end{cases}
$$

The "sufficiency" is proved.

To prove the "necessity" suppose that (4.16) does not hold. The left side of (4.16) can be written as

$$
\left(\bigcap_{\delta \in \mathbb{R}_{+}^{*}} C l\left({ }_{+} K_{\delta} \cup{ }_{-} K_{\delta}\right)\right) \bigcap\left(\bigcap_{\delta \in \mathbb{R}_{+}^{*}} C l\left({ }_{1} K_{\delta}\right)\right) .
$$

Consequently there is $c_{0} \in(0, \infty)$ such that $c_{0} \neq 1$ and

$$
c_{0} \in \bigcap_{\delta \in \mathbb{R}_{+}^{*}} C l\left({ }_{+} K_{\delta} \cup{ }_{-} K_{\delta}\right) \quad \text { and } \quad c_{0} \in \bigcap_{\delta \in \mathbb{R}_{+}^{*}} C l\left({ }_{1} K_{\delta}\right) .
$$

Hence there are sequences

$$
\left(\left(z_{n}, w_{n}\right)\right)_{n \in \mathbb{N}} \subset\left({ }_{+1} R_{p, X}^{2} \cup_{-1} R_{p, X}^{2}\right)
$$


and

$$
\left(\left(q_{n}, t_{n}\right)\right)_{n \in \mathbb{N}} \subset\left(S p^{2}(X)\right) \backslash\left({ }_{+1} R_{p, X}^{2} \cup_{-1} R_{p, X}^{2}\right)
$$

such that

$$
\lim _{n \rightarrow \infty} q_{n}=\lim _{n \rightarrow \infty} t_{n}=\lim _{n \rightarrow \infty} z_{n}=\lim _{n \rightarrow \infty} w_{n}=\infty
$$

and

$$
\lim _{n \rightarrow \infty} \frac{q_{n}}{t_{n}}=\lim _{n \rightarrow \infty} \frac{z_{n}}{w_{n}}=c_{0} .
$$

Since $c_{0} \neq 1$, there is $\varepsilon_{0}>0$ such that the inequalities

$$
\left|\frac{q_{n}}{t_{n}}-1\right| \geq \varepsilon_{0} \quad \text { and } \quad\left|\frac{z_{n}}{w_{n}}-1\right| \geq \varepsilon_{0}
$$

hold if $n$ is sufficiently large. Using (4.14) and (4.25), (4.26) we obtain

$$
\lim _{n \rightarrow \infty} \frac{\Delta\left(S\left(p, q_{n}\right), S\left(p, t_{n}\right)\right)}{\left|q_{n}-t_{n}\right|}=\frac{1+c_{0}}{\left|1-c_{0}\right|}
$$

and

$$
\lim _{n \rightarrow \infty} \frac{\Delta\left(S\left(p, z_{n}\right), S\left(p, w_{n}\right)\right)}{\left|z_{n}-w_{n}\right|}=1 .
$$

Let $n_{0} \in \mathbb{N}$ be a number such that (4.29) holds for every $n \geq n_{0}$. Define a sequence $\left(\left(s_{n}, y_{n}\right)\right)_{n \in \mathbb{N}}$ as a "mixture" of the sequences $\left(\left(q_{n}, t_{n}\right)\right)_{n \in \mathbb{N}}$ and $\left(\left(z_{n}, w_{n}\right)\right)_{n \in \mathbb{N}}$,

$$
\left(s_{n}, y_{n}\right)= \begin{cases}\left(q_{n_{0}}, t_{n_{0}}\right) & \text { if } n \leq n_{0} \\ \left(q_{n}, t_{n}\right) & \text { if } n \text { is add and } n>n_{0} \\ \left(z_{n}, w_{n}\right) & \text { if } n \text { is even and } n>n_{0}\end{cases}
$$

Then $\left(\left(s_{n}, y_{n}\right)\right)_{n \in \mathbb{N}} \subset S_{\varepsilon_{0}}^{2}$ and, by (4.27), (4.28), we have

$$
\lim _{n \rightarrow \infty} s_{n}=\lim _{n \rightarrow \infty} y_{n}=\infty, \quad \lim _{n \rightarrow \infty} \frac{s_{n}}{y_{n}}=c_{0} .
$$

If there is

$$
\lim _{n \rightarrow \infty} \frac{\Delta\left(S\left(p, s_{n}\right), S\left(p, y_{n}\right)\right)}{\left|s_{n}-y_{n}\right|},
$$

then the definition of the sequence $\left(\left(s_{n}, y_{n}\right)\right)_{n \in \mathbb{N}}$ and equalities (4.30), (4.31) imply

$$
1=\frac{1+c_{0}}{\left|1-c_{0}\right|} .
$$

Hence $c_{0}=0$, contrary to the condition $c_{0} \notin\{0,1, \infty\}$.

Thus condition (ii) from Theorem 2.1 is false if (4.16) is false. 
The following theorem is the main result of the present section. Define, for $\delta>0$,

$$
{ }_{+-} K_{\delta}:=\left\{\frac{q}{t}: q \in{ }_{+1} R_{p, X} \cap[\delta, \infty), \quad t \in{ }_{-1} R_{p, X} \cap[\delta, \infty)\right\},
$$

where $\frac{q}{t}:=\infty$ if ${ }_{-1} R_{p, X} \cap[\delta, \infty)=\varnothing$ and $\frac{q}{t}:=0$ if ${ }_{+1} R_{p, X} \cap[\delta, \infty)=\varnothing$.

Theorem 4.1. Let $X$ be an infinite subset of $\mathbb{R}$, let $d(x, y)=|x-y|$ for all $x, y \in X$ and let $p$ be a point of $X$. Then $(X, d) \in \mathfrak{U}$ if and only if $X$ is asymmetric at infinity with respect to $p$ and

$$
\bigcap_{\delta \in \mathbb{R}_{+}^{*}}\left(C l\left(_{+} K_{\delta} \cup{ }_{-} K_{\delta}\right) \cap C l\left({ }_{+-} K_{\delta}\right)\right) \subseteq\{0, \infty\} .
$$

Proof. Suppose $(X, d) \in \mathfrak{U}$. Then conditions $(i)$ and (ii) from Theorem 2.1 holds. Using Corollary 4.1 we see that $X$ is asymmetric at infinity w.r.t. $p$. Moreover, since ${ }_{1} K_{\delta} \supseteq{ }_{+-} K_{\delta}$, we obtain from inclusion (4.16) the inclusion

$$
\bigcap_{\delta \in \mathbb{R}_{+}^{*}}\left(C l\left({ }_{+} K_{\delta} \cup{ }_{-} K_{\delta}\right) \cap C l\left(_{+-} K_{\delta}\right)\right) \subseteq\{0,1, \infty\} .
$$

It still remains to prove that

$$
1 \notin \bigcap_{\delta \in \mathbb{R}_{+}^{*}}\left(C l\left({ }_{+} K_{\delta} \cup{ }_{-} K_{\delta}\right) \cap C l\left({ }_{+-} K_{\delta}\right)\right) .
$$

Statement (4.34) holds if

$$
1 \notin \bigcap_{\delta \in \mathbb{R}_{+}^{*}} C l\left({ }_{+-} K_{\delta}\right) .
$$

Suppose (4.35) does not hold. Then there exist some sequences

$$
\left(x_{n}\right)_{n \in \mathbb{N}} \subset(-\infty, p] \cap X \quad \text { and } \quad\left(y_{n}\right)_{n \in \mathbb{N}} \subset[p, \infty) \cap X
$$

such that

$$
\lim _{n \rightarrow \infty}\left|x_{n}-p\right|=\lim _{n \rightarrow \infty}\left|y_{n}-p\right|=\infty
$$

and

$$
\lim _{n \rightarrow \infty} \frac{p-x_{n}}{y_{n}-p}=1 .
$$

By Lemma 4.1 it contradicts condition (i) from Theorem 2.1. Thus (4.34) is proved. 
Conversely, assume inclusion (4.33) holds and $X$ is asymmetric at infinity w.r.t. $p$. We must prove conditions $(i)$ and $(i i)$ from Theorem 2.1.

Rewriting Lemma 4.1 in the terms of cluster sets we see that condition $(i)$ does not hold if and only if

$$
1 \in \bigcap_{\delta \in \mathbb{R}_{+}^{*}} C l\left({ }_{+-} K_{\delta}\right)
$$

This relation and

$$
1 \in \bigcap_{\delta \in \mathbb{R}_{+}^{*}} C l\left({ }_{+} K_{\delta} \cup{ }_{-} K_{\delta}\right)
$$

show that the point 1 belongs to the set in the left part of formula (4.33), contrary to the supposition. Condition $(i)$ follows.

To prove condition $(i i)$ from Theorem 2.1 note that

$$
{ }_{1} K_{\delta}={ }_{+-} K_{\delta} \cup-+K_{\delta}
$$

for sufficiently large $\delta>0$ because $X$ is asymmetric at infinity w.r.t. $p$, where ${ }_{1} K_{\delta}$ is defined by (4.15) and ${ }_{-+} K_{\delta}$ is the set obtained by the permutation of the symbols "+" and "-" in (4.32). Consequently, by Proposition 4.1, condition ( $i i$ ) holds if

$$
\bigcap_{\delta \in \mathbb{R}_{+}^{*}}\left(C l\left({ }_{+} K_{\delta} \cup{ }_{-} K_{\delta}\right) \cap C l\left({ }_{+-} K_{\delta} \cup{ }_{-+} K_{\delta}\right)\right) \subseteq\{0,1, \infty\} .
$$

Similarly (4.20) we can show that

$$
\begin{aligned}
\bigcap_{\delta \in \mathbb{R}_{+}^{*}}\left(C l\left(_{+} K_{\delta} \cup{ }_{-} K_{\delta}\right)\right. & \left.\cap C l\left(_{+-} K_{\delta} \cup{ }_{-+} K_{\delta}\right)\right) \\
= & \left(\bigcap_{\delta \in \mathbb{R}_{+}^{*}}\left(C l\left(_{+} K_{\delta} \cup{ }_{-} K_{\delta}\right) \cap C l\left(_{+-} K_{\delta}\right)\right)\right) \\
\cup & \left(\bigcap_{\delta \in \mathbb{R}_{+}^{*}}\left(C l\left(_{+} K_{\delta} \cup{ }_{-} K_{\delta}\right) \cap C l\left({ }_{-+} K_{\delta}\right)\right)\right) .
\end{aligned}
$$

It follows from the definition of the sets ${ }_{+} K_{\delta},{ }_{-} K_{\delta},{ }_{+-} K_{\delta}$ and ${ }_{-+} K_{\delta}$ that if a positive number $s$ belongs to

$$
\bigcap_{\delta \in \mathbb{R}_{+}^{*}}\left(C l\left({ }_{+} K_{\delta} \cup{ }_{-} K_{\delta}\right) \cap C l\left(-{ }_{+} K_{\delta}\right)\right)
$$

then

$$
\frac{1}{s} \in \bigcap_{\delta \in \mathbb{R}_{+}^{*}}\left(C l\left({ }_{+} K_{\delta} \cup{ }_{-} K_{\delta}\right) \cap C l\left(_{+-} K_{\delta}\right)\right) .
$$


Consequently, we can permute "+" and "-" (4.33). Thus we have the inclusion

$$
\bigcap_{\delta \in \mathbb{R}_{+}^{*}}\left(C l\left({ }_{+} K_{\delta} \cup{ }_{-} K_{\delta}\right) \cap C l\left({ }_{+-} K_{\delta} \cup-_{+} K_{\delta}\right)\right) \subseteq\{0, \infty\},
$$

which implies (4.37).

\section{References}

[1] F. Abdullayev, O. Dovgoshey, M. Küçükaslan, Metric spaces with unique pretangent spaces. Conditions of the uniqueness // Ann. Acad. Sci. Fenn. Math., 36 (2011), No. 2, 353-392.

[2] M. Altinok, O. Dovgoshey, M. Küçükaslan, Local one-sided porosity and pretangent spaces // Analysis, München, 36 (2016), No. 3, 147-171.

[3] M. Berger, Geometry, I, Corrected Fourth Printing, Springer- Verlag, Berlin Heidelberg, 2009.

[4] V. Bilet, O. Dovgoshey, Asymptotic behavior of metric spaces at infinity // Dopov. Nac. acad. nauk Ukr., (2017), No. 9, 9-14.

[5] V. Bilet, O. Dovgoshey, Finite asymptotic clusters of metric spaces // Theory and Applications of Graphs, 5 (2018), No. 2, 2-33.

[6] V. Bilet, O. Dovgoshey, Finite spaces pretangent to metric spaces at infinity // Ukrainian Mathematical Bulletin, 15 (2018), No. 4, 448-474.

[7] N. Bourbaki, Elements of Mathematics. General Topology. Chapters 5-10, Springer-Verlag, Berlin, 1998.

[8] E. F. Collingwood, A. J. Lohwater, The Theory of Cluster Sets, Cambridge Univ. Press, Cambridge, 1966.

[9] J. L. Kelley, General Topology, D. Van Nostrand Company, Princeton, 1965.

[10] L. S. Pontryagin, Selected Works, vol. 2, Topological Groups, Classics of Soviet Mathematics, Edited and with a preface by R. V. Gamkrelidze, CRC Press, 1987.

[11] M. Ó. Searcóid, Metric Spaces, Springer-Verlag, London, 2007.

[12] B. S. Thomson, Symmetric Properties of Real Functions, Marcel Dekker Inc., New York, 1994.

\section{CONTACT INFORMATION}

Oleksiy Dovgoshey Institute of Applied Mathematics and Mechanics of the NASU, Slov'yansk, Ukraine E-Mail: oleksiy.dovgoshey@gmail.com

Viktoriia Bilet

Institute of Applied Mathematics and Mechanics of the NASU, Slov'yansk, Ukraine E-Mail: viktoriiabilet@gmail.com 